Full Paper

\title{
Die Auswirkungen unterschiedlicher Standorte auf die Trauben- und Weinqualität bei der Sorte Blauburgunder
}

The effects of location on the quality of grapes and wine of the variety Pinot Noir

L'impatto di diversi siti sulla qualità dell'uva e del vino nella varietà Pinot Nero

Ulrich Pedri ${ }^{1}$, Günther Pertoll ${ }^{1}$, Martin Thalheimer ${ }^{1}$, Eva Überegger ${ }^{1}$

${ }^{1}$ Versuchszentrum Laimburg

\section{ABSTRACT}

In the context of a large-scale project investigating interdependencies between the grapevine variety and the location site of vineyard, the habitat requirements of the variety Pinot Noir was examined. Differences and similarities of different vineyard sites in the winegrowing area Überetsch (South Tyrol, Italy) and correlations between the conditions of the sites, the analytical and sensorial properties of wines from the variety Pinot Noir was investigated. Statistically significant differences could be found between the vineyard sites and also between the wines from different vineyard sites. In order to explain these relationships, it turned out that climatological factors showed more impact than pedological factors. Lightly alkaline habitats at 450 a.s.l. (above sea level), where the planted vine grapes showed a moderate growth, seemed to be an advantage for the wine quality.

\section{KEYWORDS}

Pinot Noir, Vineyard site, Soil, Climate, Wine quality

\section{CITE ARTICLE AS}

Pedri Ulrich, Pertoll Günther, Thalheimer Martin et.al. (2018). The effects of location on the quality of grapes and wine of the variety Pinot Noir. Laimburg Journal 01/2019

DOI: $10.23796 /$ LJ/2019.009

\section{CORRESPONDING AUTHOR}

Ulrich Pedri

Laimburg 6, Pfatten, I-39040 Auer

(BZ), Italien

ulrich.pedri@laimburg.it

+390471969624 


\section{EINLEITUNG}

Der Pinot Noir wird in Südtirol Blauburgunder genannt. Aktuell besetzt Blauburgunder in Südtirol eine Fläche von 389 ha bei einer Gesamtrebfläche von 5347 ha [1]. Das entspricht einem Anteil von 7.3\%. Pinot Noir gilt als Sorte von Weltniveau. Die bevorzugte Verbreitung der höchsten Qualitäten liegt in den kühlen bis schwach mittelmäßig temperierten Weinbaugebieten mit Durchschnittstemperaturen von $14.0-16.0{ }^{\circ} \mathrm{C}$ zwischen April und Oktober [2]. Das Hauptanbaugebiet liegt nach wie vor in Frankreich mit 29576 ha [3], davon vor allem in der Champagne, wo er als Sektgrundwein entweder sortenrein (blanc de noir) oder im Verschnitt eingesetzt wird. Weiters wird er in der Côted'Or (Burgund) mit 6579 ha angebaut, und man findet inn in der Côte Chalonnaise und Mâconnais oder im Elsass. Außerdem findet man Blauburgunder in Europa noch in Deutschland (Spätburgunder) im Jahr 2014 mit 11783 ha [4], in Österreich im Jahr 2009 mit ca. 649 ha [5], in der Schweiz (u.a. Syn. Clevner) im Jahr 2014 mit ca. 4260 ha [6] und in Italien mit ca. 3314 ha [7]. Außerhalb Europas wird er in Neuseeland im Jahr 2018 mit 5653 ha [8], in den USA mit 17000 ha, in Australien mit 4490 ha, außerdem in Kanada, Südafrika, Chile, Ungarn und Moldawien [3] angebaut. In Italien befindet sich Pinot Noir vor allem in Südtirol mit 389 ha [1] und in der Zone des Oltrepò Pavese mit ca. 3000 ha [9]. In Südtirol wurde Blauburgunder zwischen der Mitte und dem Ende des 19. Jh. eingeführt [10].

Zur Zeit hat er in Südtirol in einem begrenzten Gebiet in Mazzon bei Neumarkt seine größte Anbaudichte, wo er laut Dipoli und Carlotto (2009) [10] eine geeignete pedoklimatische und geomorphologische Situation vorfindet, nämlich eine Meereshöhe zwischen 350 und $400 \mathrm{~m}$ ü. NN sowie einen Geländehorizont, welcher während der Traubenreife kühle Temperaturen in den Morgenstunden ermöglicht und wo der Sonnenuntergang erst spät erfolgt. Diese mesoklimatische Situation und die Böden, auf den kalkreichen Sedimenten der Trias durch mehr oder weniger starke fluvioglaziale $A b$ lagerungen der letzten Eiszeit, verleihen den Autoren nach den Blauburgunder-Weinen dieser Zone ihre besondere Güte.

Vereinzelte kleinere Flächen mit Blauburgunder befinden sich in ganz Südtirol verteilt auf einer Meereshöhe zwischen 300 und $800 \mathrm{~m}$ auf sehr unterschiedlichen Böden, von sehr kalkhaltigen bis zu sauren Bodenreaktionen. Entsprechend der weltweiten Verbreitung ist Pinot Noir Objekt zahlreicher Studien verschiedenster Fragestellungen. Die Studien haben unter anderem die Zusammensetzung der Trauben und Weine aus Blauburgunder im Allgemeinen als Fokus, zumal die Zusammensetzung für die Wahrnehmung der Weine durch den Konsumenten von Bedeutung ist. So untersuchte Wenzel et al. (1987) [11] die Anthocyanzusammensetzung der Blauburgunder-Beeren und stellte fest, dass diese Sorte keine acylierten Anthocyane aufweist und sich somit deutlich von dem Großteil anderen Rotweinrebsorten unterscheidet.

Auch die Standortfrage, besonders in $\mathrm{Zu}$ sammenhang mit dem Pinot Noir, wurde öfters untersucht und diskutiert. In Frankreich und speziell im Burgund ist das Konzept des "terroir" oder "climats" sehr eng mit der Weinqualität verbunden [12], [13], [14]. Dementsprechend zahlreich sind die Studien, welche sich damit befassen [15], [16], [17], [18]. Mériaux et al. (1981) [15] versuchte für die Côte-d'Or anhand einer Formel das Qualitätsniveau der Lagen zu berechnen. Die Formel berücksichtigt die Hangneigung, den Steinanteil, die Durchwurzelungstiefe, den Kalkgehalt und Tonanteil des Feinbodens und die Menge an austauschbarem Kalium. Die Berechnung führte zu einem topo-pedologischen Index, der in den Grand Cru Lagen des Burgunds stets höher lag als in den Village oder Bourgogne-Lagen. Seguin (1986) [17] spricht weiter von der wichtigen Bedeutung der Bodenstruktur, wichtiger als etwa der Tonanteil und Skelettanteil. Für van Leeuwen und Seguin (2006) [18] liegen im Burgund die besten Lagen etwa bei $300 \mathrm{~m}$ ü. NN. Ausschlaggebend dafür ist ein langsamer, aber zur Vollendung gebrachter Reifeprozess. Die optimale Standort-Sorten-Kombination ermöglicht der Sorte eine möglichst langsame aber stete Reife. Nur dann kann das aromatische Potential von Sorte und Standort voll ausgeschöpft werden [19]. Laut Sittler und Marocke (1981) [20] findet Pinot Noir im Elsass auf sandigen, kalkhaltigen Böden optimale Standorte. Es ist mittlerweile anerkannt, dass der Standort (terroir) ein System von Wechselwirkungen zwischen Boden, Geomorphologie, Klima und Mensch ist [18]. Dennoch gibt es immer wieder Versuche, die Bedeutung einzelner Standortfaktoren auf die Weinqualität zu erklären. Die komplexen Zusammenhänge zwischen dem Standort und den sensorischen Eigenschaften der Weine sind Gegenstand zahlreicher Studien, so untersuchte Cortell et al. (2008) [21] den Einfluss der Wuchsstärke einzelner Rebstöcke auf die Gerbstoffzusammensetzung von Wein und dessen Auswirkung auf die adstringierenden und bitteren Eindrücke in der Sensorik. So wurde beobachtet, dass es bei Rebstöcken unterschiedlicher Wuchsstärke gesicherte Unterschiede in der Wahrnehmung der Adstringenz gibt, und dass die Adstringenz mit abnehmender Wuchsstärke zunahm, während die Bitterkeit abnahm. Die Wuchsstärke eines Rebstockes wird maßgeblich durch die Standorteigenschaften und im Speziellen durch das Wasser- und Nähstoffspeichervermögen des Standortes beeinflusst. Als Maßzahl für die Wuchsstärke wird international der Ravaz-Index herangezogen [22]. Durch die Wuchsstärke der Rebanlage wird das Mikroklima in der Laubwand beeinflusst. Insofern kann getrost behauptet werden, dass die Standorteigenschaften, welche vom Boden kommen, das Mikroklima in der Traubenzone und somit die Traubenzusammensetzung prägen [23].

Mit dem Lagenkonzept rund um die Sorte Pinot Noir im Burgund wird auch der Begriff "climat" als Abgrenzung eines Standortes oder einer Lage verwendet, so dass sich daraus auch die Bedeutung des Klimas ableiten lässt [13].

Die Bedeutung von Sonneneinstrahlung und Temperatur zur Farbausprägung wird in der Literatur mehrfach erwähnt [23], [24], wobei es durchwegs auch sortenspezifische Unterschiede gibt. Pinot Noir reagiert bei höherer Sonnenexposition der Trauben mit stärkerer Einlagerung an Gesamtanthocyanen, während der Gehalt an Catechin und Epicatechin dadurch sinkt. Diese beiden chemischen Gruppen sind von besonderer sensorischer Bedeutung [21].

In Zusammenhang mit dem Einfluss von Temperatur und Sonneneinstrahlung ist der Jahrgangseinfluss zu sehen und dessen Auswirkung auf die Polyphenolzusammensetzung [24].

In Südtirol selbst sind die Temperaturschwankungen von Jahr zu Jahr erheblich, somit konnte ein deutlicher Jahrgangseffekt zum Beispiel bei Sauvignon Blanc im Südtiroler Überetsch beobachtet werden [25].

Der größte Teil des Weinbaues in Südtirol befindet sich in einer Meereshöhe zwischen $220 \mathrm{~m}$ und $1000 \mathrm{~m}$ ü. NN in einer feuchttemperierten Klimate des Typs "Cfa" (Feuchttemperierte Klimate mit einer mittleren Temperatur des wärmsten Monats $>22^{\circ} \mathrm{C}$ ) 
nach Köppen (1918) [26] und kann umgangssprachlich als kontinental-inneralpines Klima bezeichnet werden. Die in der vorliegenden Arbeit beschriebene Sorten-LagenStudie findet im Südtiroler Überetsch, zwischen $247 \mathrm{~m}$ und $572 \mathrm{~m}$ ü. NN, unter den oben genannten klimatischen Bedingungen statt.

\section{MATERIAL UND METHODEN}

\section{BODEN UND KLIMA}

Die Basis für die Auswahl der Standorte wurde auf Grund der Kartierung des untersuchten Gebietes nach Thalheimer (2006) [27] vorgenommen. Die untersuchten Standorte (Tab. 1 und Tab. 2) entsprechen jenen aus Pedri und Pertoll (2012) [25]. Es wird darauf verwiesen und die Eigenschaften im Einzelnen im vorliegenden Artikel nicht mehr detailliert diskutiert.

Die Wärmesummenindizes der beobachteten Standorte nach Huglin (1978) [28] liegen im Schnitt der Jahre 1996-2002 zwischen einem Wert von 1869 (576 m ü. NN) und einem Wert von 2329 (247 m ü. NN) (Tab. 3).

Maaß und Schwab (2012) [29] klassifizieren z.B. den Standort Laimburg (220 m ü. NN) mit seinem dekadenmittleren (2001-2010) Huglin-Index von ca. 2350 als "warm". Für Seguin und Garcia de Cortazar (2005) [30] kennzeichnen Werte zwischen 1800 und 2100 "gemäßigte" Standorte, jene zwischen 2100 und 2400 "gemäßigt heiße" (warme) Standorte. Es werden demzufolge also sechs von acht geprüften Standorten im Südtiroler Überetsch der Kategorie "gemäßigt heiß" (warm) zugeordnet und zwei von acht jener der "gemäßigten" Standorte. Der Huglin-Index ist im beobachteten Gebiet größtenteils vor allem von der Meereshöhe abhängig, wobei der Wert mit zunehmender Meereshöhe abnimmt. Die Grenze zwischen einem "gemäßigten" und "gemäßigt-heißen" Standort liegt im Südtiroler Überetsch bei ca. $470 \mathrm{~m}$ ü. NN.

Für Huglin (1978) [28] selbst liegt der HuglinWärmesummenindex für Blauburgunder bei 1700. Nach Petgen (2007) [31] sind die Bedürfnisse jenen von Chardonnay und Sauvignon gleichzusetzen. Maaß und Schwab (2011) [32] passen die Huglin-Werte für kühlere Weinbauklimate an und setzen den Wert für Blauburgunder auf 1700 und somit vergleichbar mit Sauvignon aber niedriger als Chardonnay an.

\section{WEINBAULICHE ERHEBUNGEN}

Die weinbaulichen Erhebungen wurden von 1996-2002 durchgeführt.

Jährlich wurden ab Ende März von jeder Versuchsanlage die phänologischen Entwicklungsstadien nach der BBCH-Skala [33] aufgezeichnet. Somit kann der zeitliche Ablauf von Austrieb, Blüte, Reifebeginn und Reife $\left(16^{\circ} \mathrm{KMW}\right)$ rückverfolgt werden.

Ab Weichwerden der Beeren (BBCH 81) wurden in wöchentlichem Abstand Reifeproben gezogen und der Reifeverlauf für die jeweilige Lage erstellt. Bei den Analysen wurden der Zuckergehalt in Klosterneuburger Mostwaage $\left({ }^{\circ} \mathrm{KMW}\right)$, die titrierbare Gesamtsäure $(\mathrm{g} / \mathrm{l})$ und der $\mathrm{pH}$-Wert ermittelt. Auf diese Weise konnte ein zuverlässiges Bild über den Reifegrad der Trauben gewonnen und somit der optimale Zeitpunkt für die Ernte der jeweiligen Lage festgesetzt werden.

Weiters wurden die Anzahl der Trauben und Triebe pro Stock, die Erträge (Ertrag pro Stock, Ertrag pro $\mathrm{m}^{2}$ ) und das mittlere Traubengewicht erhoben. In den Jahren 19961999 wurden zur Traubenlese Erhebungen zum Befall durch Botrytis cinerea und Essigfäule durchgeführt. Dabei wurde mittels visueller Bonitur der Prozentsatz der Befallstärke der Trauben pro Stock ermittelt.

Beim Rebschnitt wurde das Schnittholzgewicht (Gewicht des einjährigen Holzes) erhoben und der Ravaz-Index [22] errechnet. Dieser ergibt sich aus dem Verhältnis zwischen Ertrag und Schnittholz und kann mit dem Blatt-Frucht-Verhältnis verglichen werden. Um einen Einfluss des Ertragsniveaus auf die Weinbereitung möglichst gering zu halten, wurde eine Ertragsregulierung durchgeführt.

Der Reifeverlauf wurde durch wöchentliche Probenahmen von 150 Beeren je Standort durchgeführt. Diese Beerenproben wurden händisch gequetscht, mittels einer kleinen Labormembranpresse zweimal für je 1 min. bei 2 bar gepresst, der Most zentrifugiert und gesiebt. Es erfolgten Messungen des Mostgewichtes ('KMW) (refraktometrisch), der titrierbaren Gesamtsäure (g/L) (Reg CEE 2676/90 All.pto 13) und des pH-Wertes (Reg CEE 2676/90 All.pto 24).

\section{WEINBEREITUNG, SENSORISCHE UND CHEMISCHE ANALYSEN}

Die Weine wurden gemäß folgendem Standardprotokoll ausgebaut, analysiert und verkostet:
- Die Trauben wurden mittels einer Abbeermaschine des Typs Zambelli abgebeert und gequetscht.

- $28 \mathrm{~kg}$ Maische wurden in Weithalsglasballons gefüllt und mit Trockenreinzuchthefepräparat der Bezeichnung Levuline BRG nach vorhergehender Rehydrierung geimpft.

- Der Schalenkontakt erfolgte während der gesamten alkoholischen Gärung bis zur Durchgärung unter $2.5 \mathrm{~g} / \mathrm{l}$ Restzucker.

- Händisches Untertauchen des Tresterhutes für eine Woche einmal täglich;

- Gärung in der Gärzelle bei kontrollierter Temperatur von max. $28^{\circ} \mathrm{C}$;

- Gärungskontrolle durch regelmäßige Restzuckeranalysen und Abstich bei Restzucker $\leq 2.5 \mathrm{~g} / \mathrm{L}$ );

- erster Abstich und die Beigabe von Druckmost ( 0.5 bar) ohne Zugabe von schwefeliger Säure;

- der zweite Abstich zwei Wochen nach Gärende;

- Lagerung in der Gärzelle bei Temperatur von $20^{\circ} \mathrm{C}$ bis zum Ende der malolaktischen Gärung (BSA).

Die Weinausbauten erfolgten 1996 bis 1999. Die Weine wurden dreimal (Jahrgänge 1997 und 1998) oder viermal (Jahrgänge 1996 und 1999) jeweils im Jahresabstand verkostet, wobei die absolut letzte Verkostung 2005 stattfand. Dafür wurde eigens eine Verkostungskommission trainiert. Das Panel setzte sich aus Technikern des Versuchszentrums Laimburg, Weinbauberatern und Kellermeistern aus Südtirol zusammen. Für die Schulung wurden Fassproben, gefüllte Weine aus Praxisbetrieben und Versuchsweine verwendet.

Die Verkostungskommission wurde in zwei Gruppen eingeteilt und jeder Gruppe wurden alle Weine anonym in einer anderen randomisierten Reihenfolge als Einzelproben gereicht. Es wurden 20 einzelne Muster beurteilt, wobei einige Weine mehrmals anonym gereicht wurden. Diese doppelt gereichten Weine dienten zur Feststellung der Urteilssicherheit. Als Beurteilungsschemata wurden für jede Sorte eigene Profilanalysen nach Weiss (1972) [34], modifiziert angefertigt. Die gefragten Parameter waren sortenspezifisch und a priori anhand der durchgeführten Schulungsverkostungen festgelegt. Für die Endauswertung wurden nur jene 
Koster berücksichtigt, welche als urteilssicher galten [35]. Neben der sensorischen Beurteilung der Weine wurden die wichtigsten wertgebenden Inhaltsstoffe von Most und Wein gemessen:

- Mostgewicht (refraktometrisch),

- titrierbare Gesamtsäure im Most und Wein (Reg CEE 2676/90 All.pto 13),

- $\mathrm{pH}$-Wert im Most und Wein (Reg CEE 2676/90 All.pto 24),

- Alkoholgehalt im Wein (Reg CEE 2676/90 All.pto 3 "elektronische Dichtemessung" Reg Ce 355/2005 par 4c),

- Gesamtextrakt (Reg CEE 2676/90 All.pto 4),

- zuckerfreier Extrakt im Wein (rechnerisch: Gesamttrockenextrakt - Red Zucker + 1),

- Weinsäure und Äpfelsäure im Wein (Ionenchromatographisch), Milchsäure im Wein (HPLC),

- Gesamtgerbstoffe nach Folin im Wein (Folin-Ciocalteau Methodenbuch 5.04, 17),

- hefeverwertbarer Stickstoff in den Jahren 1997 und 1998 (Ninhydrinmethode),

- Restzucker (modifizierter Rebelein-Methode gemessen, $=5.04 \mathrm{mi} 10$ rev4 2007 laut Methodenbuch der Weinchemie),

- Anthocyane (photometrisch nach Somers \& Evans).

Zur Unterscheidung der Lagen wurden die Daten varianzanalytisch verrechnet. Die statistische Verrechnung erfolgte über multivariante Varianzanalyse (MANOVA) zur Feststellung der Wechselwirkungen und schließlich über Mittelwertvergleiche (Oneway-ANOVA) um eine belastbare jahrgangs- und reifungsunabhängige Aussage treffen zu können. Dafür wurden alle Einzelwerte über eben diese Faktoren gemittelt und in Folge mit den Mittelwerten die statistische Berechnung durchgeführt. Als Statistikprogramm diente SPSS für Windows Release 11.0.1 und 12.0 von (C) SPSS Inc. 1989-2001. Weiters wurden die Lagen bezüglich ihrer Sensorik über Clusteranalysen und Hauptkomponentenanalysen gruppiert und charakterisiert. Diese deskriptive Hauptkomponentenanalyse erfolgte über The Unscramb$\operatorname{ler}^{\circledR} X$ Version 10.3 (64-bit) (C) 2009-2013 CAMO Software.

Die Zusammenhänge zwischen den einzelnen gemessenen Parametern wurden, um
Scheinkorrelationen zu vermeiden, über partielle Korrelationen gesucht (über Weinbaulage und Jahrgang).

\section{ERGEBNISSE}

\section{STANDORTE, BÖDEN UND KLIMA}

Aufgrund der Beschaffenheit des geologischen Ausgangsmaterials und der geologischen Entstehungsgeschichte der Landschaft können die Böden der Versuchsanlagen wie folgt beschrieben werden.

In den Versuchsanlagen Eppan "Berg", Kaltern "Dorf", "St. Josef am See" und Planitzing "Garnellen" befinden sich Böden auf Kalkgesteinsschutt, die im westlichen Teil des Überetsch in Form von Schwemmkegeln abgelagert wurden. Diese Böden haben einen mittleren bis hohen Skelettanteil (fast ausschließlich Dolomit- und Kalkgesteine), einen mittleren bis hohen Gehalt an Kalziumkarbonat in der Feinerde $(\mathrm{pH}$-Werte im alkalischen Bereich), eine rötliche Farbe, sandig-lehmige bis lehmige Bodenart und eine hohe durchwurzelbare Tiefe.

Die Böden in den Versuchsanlagen Girlan "Doos", "Schreckbichl" und Kaltern "Mazzon" sind Moränenablagerungen, die sich durch das Geschiebe aus grobem und feinem Gesteinsmaterial der Gletscherströme gebildet haben. Diese Böden stellen flächenmäßig den größten Anteil der landwirtschaftlich genutzten Böden im Überetsch dar und charakterisieren das Landschaftsbild durch die lang gezogenen stromlinienförmigen Hügel. Deutlich sichtbar ist in diesen Böden ein Verwitterungshorizont, der durch die Verwitterungslösung des Kalziumkarbonats und die darauffolgende Verbraunung und Versauerung entstand. Es handelt sich um durchwegs leicht saure Böden.

Die Böden auf späteiszeitlichen Seenablagerungen (Versuchsanlage St. Pauls "Feld") sind hauptsächlich im nördlichen Überetsch anzufinden. Sie sind sandig-lehmig, sauer, wasserdurchlässig, sehr gut durchwurzelbar und andererseits von einem bescheidenen Wasser- und Nährstoffhaltevermögen gekennzeichnet. Der Humusgehalt ist durchwegs sehr niedrig und erreicht selten $2 \%$.

In Tabelle 2 sind die Mittelwerte der Lufttemperatur auf $2 \mathrm{~m}$ Höhe, der Bodentemperatur in $50 \mathrm{~cm}$ Tiefe, der relativen Luftfeuchtigkeit, des Niederschlags, der Windgeschwindigkeit und Windrichtung von den verschiedenen Versuchsanlagen von 1996-
2002 aufgezeichnet. Die höher gelegenen Lagen Eppan "Berg" und Planitzing "Garnellen" zeigen die niedrigsten Jahresdurchschnittstemperaturen auf, während die Standorte "St. Josef am See" und Kaltern "Mazzon" die wärmsten Lagen sind.

Bei der Bodentemperatur in $50 \mathrm{~cm}$ Tiefe wurde im lehmigen Sand in St. Pauls "Feld" der tiefste Wert registriert. Die höchsten Werte sind wiederum in "St. Josef am See" und Kaltern "Mazzon" zu verzeichnen.

Trotz der geringen Entfernungen zwischen den Versuchsanlagen (max. ca. $10 \mathrm{~km}$ ), zeigten sich in jenen der Gemeinde Kaltern mehr Niederschläge im Gegensatz zu jenen in der Gemeinde Eppan.

Luftige Standorte mit durchschnittlichen Windgeschwindigkeiten von $1.79 \mathrm{~m} / \mathrm{s}, 1.44$ $\mathrm{m} / \mathrm{s}$ und $1.23 \mathrm{~m} / \mathrm{s}$ sind jeweils "Schreckbichl", Kaltern "Mazzon" und "St. Josef am See". Die Hauptwindrichtung im Überetsch ist Süd-Südwest oder Süd-Südost.

Der Huglin-Index der acht Versuchsanlagen zwischen 1996 und 2002 ist in Tabelle 3 dargestellt.

Er lag in den untersuchten Standorten in diesem Zeitraum zwischen 1833 (Eppan Berg im Jahr 1996) und 2505 (St. Josef am See 1998). Den im Schnitt der Jahre tiefsten Huglin-Indexwert zeigte erwartungsgemäß der höchstgelegenste Standort Eppan Berg mit 1869. Somit wurden der Mindestwert nach Huglin oder Maaß und Schwab (2011) [32] von 1700 auch am höchstgelegenen Standort überschritten.

Die Eigenschaften der Standorte wirkten sich auch auf das Rebwachstum und die Traubengröße (Tab. 4) aus. Obwohl eine Ertragsregulierung durchgeführt wurde, konnte man statistisch signifikante Unterschiede in der Ertragshöhe finden. Die Ursache für diese Ertragsunterschiede trotz $\mathrm{Er}$ tragsregulierung sind hauptsächlich im unterschiedlichen mittleren Traubengewicht zu finden (Abb. 1). Das mittlere Traubengewicht schwankte von $132.4 \mathrm{~g}$ am Standort Kaltern "Dorf" bis $159.1 \mathrm{~g}$ am Standort Girlan "Schreckbichl". Höchstsignifikante Unterschiede wurden auch bezüglich des Schnittholzgewichtes, des Ravaz-Indexes und der Botrytis-Befallsstärke gefunden. In besonderem Maße ist die Botrytis-Befallsstärke von Bedeutung, da ein Botrytis-Befall entweder zu einem spürbaren Ertragsausfall oder zur Schmälerung der Maischequalität führt. Aus diesem Grund sind Produzenten 
in Botrytis-sensiblen Standorten häufig gezwungen ihre Trauben bei beginnendem Botrytis-Befall früher als zum optimalen Zeitpunkt zu lesen. So zeigte sich der tiefste Standort Kaltern "St. Josef am See" (247 m ü. NN) in besonderem Maße anfällig für den Ausbruch von Botrytis-Infektionen (ca. 25\% Botrytis-Befallsstärke). Der fortgeschrittene Reifezustand bei gleichzeitig warmer Witterung im August und jahrgangsbedingt auftretenden Regenphasen führt vielfach dazu, dass die Trauben der naturgemäß engbeerigen Sorte Blauburgunder häufig im Traubeninneren feucht bleiben und so optimale Bedingungen für den Botrytis-Pilz vorliegen. Am Standort mit dem schwächsten Rebenwachstum, gemessen am Schnittholzgewicht, Kaltern "Mazzon", war im Schnitt der Jahre der Botrytis-Befall am geringsten (Abb. 2).

Die Tabelle 5 zeigt die einzelnen Daten zu den phänologischen Stadien, darunter auch das Weichwerden der Beeren. Dieses Stadium fällt je nach Standort auf unterschiedliche Zeitpunkte. Zwischen dem tiefsten und dem höchsten Standort liegen im Schnitt der Jahre knapp 19 Tage. Das bedeutet, dass der Reifebeginn am tiefsten Standort meist auf Ende Juli und somit in eine sehr warme Zeit fällt, während derselbe phänologische Zustand am höchstgelegensten Standort bereits auf eine deutlich kühlere Zeit fällt.

Der Reifeverlauf spiegelt im Wesentlichen die Unterschiede der Standorte während der gesamten Vegetationsperiode wieder. Exemplarisch dafür ist in Abbildung $3 \mathrm{der}$ Reifeverlauf des Jahres 2002 abgebildet. Man erkennt deutlich die großen Unterschiede zwischen den Lagen zu jedem Zeitpunkt der Probenahme. Besonders die zwei Standorte Kaltern "St. Josef am See" sowie Kaltern "Mazzon" zeichnen sich durch einen sehr frühen Reifebeginn, in der dritten Julidekade aus. Am Standort Kaltern "St. Josef am See" führt der frühe Reifebeginn dazu, dass die Botrytis-Anfälligkeit deutlich ansteigt (Abb. 2).

\section{DIE WEINE}

Die im Wein messbaren Unterschiede zwischen den Standorten sind erheblich. Tabelle 6 stellt diesbezüglich eine Übersicht dar. Hochsignifikante Unterschiede $(* * *)$ wurden beim pH-Wert im Most, der Säure im Most, dem hefeverwertbaren Stickstoff, den Gesamtpolyphenolen (Abb. 4) sowie dem sensorisch wahrnehmbaren harten Gerbstoff festgestellt. Stark signifikante $(* *)$

\section{ZUSAMMENFASSUNG}

Im Rahmen eines groß angelegten Sorten-Lagen-Projektes wurden die Standortansprüche der Sorte Blauburgunder (Pinot Noir) untersucht. Es wurden im Weinbaugebiet Überetsch (Südtirol, Italien) Unterschiede und Gemeinsamkeiten verschiedener Standorte untersucht und Zusammenhänge zwischen den Standortbedingungen und den analytischen sowie sensorischen Eigenschaften von Weinen der Sorte Blauburgunder erforscht. Dabei konnten statistisch signifikante Unterschiede zwischen den Standorten selbst und den Weinen der verschiedenen Weinbaulagen festgestellt werden. Auf der Suche nach den Zusammenhängen zwischen den gemessenen Faktoren stellten sich klimatologische Faktoren als bedeutsamer heraus als pedologische Faktoren. Tendenziell waren leicht alkalische Standorte über 450 m ü. NN, wo die darauf gepflanzten Reben ein nur moderates Wachstum aufwiesen, von Vorteil für die Weinqualität.

\section{RIASSUNTO}

Nell'ambito di un progetto sulla vocazionalità alla coltivazione viticola condotto su un'ampia superficie si sono investigate le esigenze pedo-climatiche del vitigno Pinot nero (Pinot Noir). Nel comprensorio viticolo dell'Oltradige (Alto Adige, Italia) sono state valutate differenze e analogie rilevate in diverse località e sono state individuate relazioni specifiche tra le condizioni di queste ultime e le caratteristiche analitiche e sensoriali riscontrate nei vini di Pinot nero. È stato possibile accertare differenze statisticamente significative tra i diversi siti ed i vini ottenuti nelle diverse zone. Nella ricerca di correlazioni tra i fattori presi in considerazione è emerso che le condizioni climatiche hanno avuto un'influenza maggiore rispetto al fattore "suolo". Tendenzialmente erano i terreni leggermente alcalini, situati ad un'altitudine superiore a $450 \mathrm{~m}$ s.l.m. dove le viti prese in esame mostravano uno sviluppo moderato, a vantaggio di una produzione di qualità.

Unterschiede wurden bezüglich des Entwicklungsstadiums und der Vielseitigkeit der Weine gefunden. Signifikante Unterschiede $(*)$ im Wein konnten für die Parameter Milchsäure, pH-Wert, titrierbare Säure, Alkohol, sensorisch wahrnehmbare Fülle (dünn - voll), Gesamtqualität (kleine Qualität - gute Qualität), sensorisch wahrnehmbare Menge an Gerbstoff (zu wenig - zu viel) und der sensorischen Gerbstoffbitterkeit (nicht bitter - bitter) festgestellt werden.

Nicht signifikante Unterschiede wurden beim Mostgewicht, beim Gehalt an Weinsäure im Wein, dem Gesamtextrakt und der Typizität der Weine festgestellt. Die nicht signifikanten Unterschiede in Mostgewicht und Weinsäure sind darauf zurückzuführen, dass versucht wurde, den maximalen Ausreifungsgrad zu erreichen, somit wurde der Lesezeitpunkt an den Standort angepasst, und demzufolge näherten sich diese Messwerte einander an. Dass beim Deskriptor der Typizität kein statistisch signifikanter Unterschied zu Tage getreten ist, beschreibt im Grunde nichts anderes, als dass alle Weine in ähnlichem Maß als Pinot Noir zu erkennen waren. Am stärksten äußerte sich der Standorteinfluss bezüglich der Kriterien "Reifezustand" und der Gerbstoffkomponenten "hart bis nicht hart" und "bitter bis nicht bitter" (Abb. 5, Abb. 6 und Abb.7).

Anhand einer multifaktoriellen Datenanalyse kann man erkennen, dass die Zusammenhänge der Bodenkennwerte und erhobenen Klimadaten zu den sensorischen Eigenschaften der Weine wenig ausgeprägt sind. So konnte nur indirekt ein Einfluss des Bodens auf die Weinqualität festgestellt werden, nämlich über die Wuchsstärke der 
Rebstöcke und dessen Einfluss auf den Entwicklungszustand der Weine (Abb. 8 und Abb. 9).

In Tabelle 7 sind die Zusammenhänge zwischen den klimatischen, bodenkundlichen, weinbaulichen, weinchemischen und sensorischen Charakteristiken ersichtlich. Das Schnittholzgewicht oder der Ravaz-Index als Maß der Wuchsstärke einer Rebe korreliert in gewissem Maß mit einigen Inhaltsstoffen wie dem hefeverwertbaren Stickstoff im Most oder dem Gehalt an Gesamtpolyphenolen im Wein und den sensorischen Eigenschaften zur Wahrnehmung der Gerbstoffe. Insofern sind Standorte, die eher zu schwachwachsenden Rebanlagen führen vorteilhafter als Böden, die das Wachstum der Reben fördern.

\section{SCHLUSSFOLGERUNG}

Grundsätzlich ist die Sorte Blauburgunder für den Anbau im gesamten untersuchten Gebiet geeignet. Die Trauben reifen auch an den höchsten Standorten genügend aus. Im beobachteten Gebiet konnten eindeutige Standortunterschiede festgestellt werden.
Diese Unterschiede betreffen, bezogen auf die Standorteigenschaften, sowohl die bodenkundlichen, als auch die klimatologischen Eigenschaften, bezogen auf den Rebenanbau und Weinausbau sowohl weinbauliche, als auch önochemische und sensorische Charakteristiken der am Standort gewachsenen Reben und der daraus entstandenen Weine. Es konnten nur wenige belastbare Hinweise auf einzelne Bodenkennoder Klimadaten gefunden werden, welche unmittelbar einen Einfluss auf die Weinqualität ausüben. Tendenziell waren klimatische Einflüsse bedeutender als Einflüsse, die vom Untergrund und Boden kommen. Es scheinen die Meereshöhe, die Temperaturindizes und die Niederschlagsmenge während der Vegetationsperiode eine gewisse Rolle zuspielen. Der Anbau in den tiefgelegenen, sehr warmen Standorten war aufgrund phytosanitärer Herausforderungen problematisch. Tendenziell waren Böden mit einer leicht alkalischen Bodenreaktion vorteilhafter, als jene mit einer leicht sauren. Wie kaum eine andere Sorte verlangt die Sorte Blauburgunder eine optimale Kombination der für sie günstigsten Standortbedingungen, um die qualitativ besten Ergebnisse zu ermöglichen.
Um die detaillierten Ursachen zu untersuchen, welche schließlich in Qualitätsunterschiede der Weine münden, sind weitere gezielte Untersuchungen nötig.

Es kann für das Südtiroler Überetsch allgemein gesagt werden, dass Blauburgunder über $450 \mathrm{~m}$ ü. NN gute Ergebnisse liefert, idealerweise auf leicht alkalischen und gut dränierten Böden, die zu einem sehr moderaten Wasserstress führen, welcher das Wachstum der Reben einbremst und eine natürliche Lockerbeerigkeit der Trauben begünstigt. Nach oben stellen im Südtiroler Überetsch $580 \mathrm{~m}$ ü. NN keine klare Grenze für diese Sorte dar. 


\section{LITERATUR}

[1] Autonome Provinz Bozen (ed.) (2012). Agrarund Forstbericht 2012. Bozen, Italien. p. 84. Retrieved August 12, 2016 from http://www.provinz.bz.it/land-forstwirtschaft/landwirtschaft/agrar-forstbericht.asp.

[2] Jones G. V., White M. A., Cooper O. R., Storchmann K. (2005). Climate Change and Global Wine Quality. Climatic Change 73 (3), 319343, DOI: 10.1007/s10584-005-4704-2.

[3] Robinson J., Harding J., Vouillamoz J. (2012). Wine Grapes. A complete guide to 1368 vine varieties, including their origins and flavours. Ecco, New York, USA, pp. 808-815.

[4] Deutsches Weininstitut (2015). Deutscher Wein Statistik 2015/16, p. 9. Retrieved March 1, 2016 from http://www.deutscheweine.de/fileadmin/user upload/Website/Service/Downloads/Statistik 2015-2016.pdf.

[5] Österreich Wein (2015). Dokumentation Österreich Wein 2014, p. 5. Retrieved August 12, 2016 from https://www.oesterreichwein.at/presse-multimedia/statistik/statistik-archiv/.

[6] Schweizerische Eidgenossenschaft - Bundesamt für Landwirtschaft (BLW) (2015). Das Weinjahr 2014. Weinwirtschaftliche Statistik, p. 5. Retrieved April 1, 2016 from: https://www.blw.admin.ch/dam/blw/de/dokumente/Nachhaltige\%20Produktion/Pflanzliche\%20Produktion/Weine\%20und\%20Spirituosen/Weinwirtschaftliche\%20Statistik/2014.pdf.download.pdf/Das Weinjahr 2014 Weinwirtschafliche Statistik.pdf.

[7] Di Vecchi, M. (2016). I vitigni minori da vino in Italia. In: Di Vecchi M., Bagni M. (eds.). Atti del Convegno Internazionale "Valore e funzione dei vitigni autoctoni e tradizionali", Lastra a Signa (FI), Italy, december 10, 2002. Slow Food Scandicci, Scandicci (FI), Italia, p. 79. Retrieved April 1, 2016 from http://www.ampelis.it/3.pdf.

[8] NEW ZEALAND WINE (2018): Vineyard Register Report. New Zealand Winegrowers 20172020, p. 5. Retrieved May 4, 2019 from https://www.nzwine.com/media/12952/ vineyard-register-report-2018.pdf.

[9] ERSAF - Ente Regionale per i Servizi all'Agricoltura e alle Foreste. Retrieved March 1, 2016 from http://www.ersaf.lombardia.it/servizi buonalombardia/Prodotti fase03.aspx?lang $=1 \& \mid \mathrm{D}=810$.

[10] Dipoli P., Carlotto M. (2009). Mazon und sein Blauburgunder. Verschönerungsverein Arkadia Edition, Geilnau, Deutschland.
[11] Wenzel K., Dittrich H., Heimfarth M. (1987). Die Zusammensetzung der Anthocyane in den Beeren verschiedener Rebsorten. Vitis 26 (2), 65-78. Retrieved March 1, 2016 from https://ojs.openagrar.de/index.php/VITIS/article/view/5911.

[12] Rigaux J. (2010). Le réveil des terroirs. Défense et illustration des "climats" de Bourgogne. Editions de Bourgogne, [Messigny-etVentoux], France.

[13] Vaudour E. (2005). I Terroir. Definizioni, caratterizzazione e protezione. Edagricole, Bologna, Italia.

[14] Wilson J. E. (1999). Terroir. Schlüssel zum Wein. Boden, Klima und Kultur im französischen Weinbau. Hallwag, Stuttgart, Deutschland.

[15] Mériaux S., Chrétien J., Vermi P., Leneuf N. (1981). La côte viticole, ses sols et ses crus. Bulletin Scientifique de Bourgogne 34, 17-40

[16] Seguin G. (1983). Influence des terroir viticoles sur la constitution et la qualité des vendages. Le Bulletin de I'OIV 56 (623), 3-16.

[17] Seguin G. (1986). 'Terroirs' and pedology of wine growing. Experientia 42 (8), 861-873, DOI: 10.1007/BF01941763.

[18] Van Leeuwen C., Seguin G. (2006). The concept of terroir in viticulture. Journal of Wine Research 17 (1), 1-10, DOI: 10.1080/09571260600633135.

[19] Ribereau-Gayon J., Peynaud E. (1971). Trattato di Enologia Vol. I. Maturazione dell'uva fermentazione alcoolica vinificazione. Edagricole, Bologna, Italia, p. 131.

[20] Sittler C., Marocke R. (1981). Geologie et oenologie en Alsace. Sols et terroirs geologiques, cepages et specificite des vins. Sciences géologiques. Bulletin et mémoires 34 (3), pp. 147-182. Retrieved March 1, 2016 from https://www.persee.fr/doc/sgeol 03022692 1981_num 34_3 1598.

[21] Cortell J. M., Sivertsen H. K., Kennedy J. A., Heymann H. (2008). Influence of Vine Vigor on Pinot Noir Fruit Composition, Wine Chemical Analysis, and Wine Sensory Attributes. American Journal of Enology and Viticulture 59 (1), 1-10.

[22] Ravaz L. (1906). Influence de la surproductionsur la vègètation de la vigne. Coulet, Montpellier, France.

[23] Price S. F., Breen P. J., Valladao M., Watson B. T. (1995). Cluster Sun Exposure and Quercetin in Pinot Noir Grapes and Wine. American Journal of Enology and Viticulture 46 (2) 187-194.

[24] Bertamini M., Mattivi F., Nicolini G. (1998). L'influenza del clima e delle tecniche di gestione del vigneto sui polifenoli del vino.
L'Enotecnico 34 (10), 31-42. http://hdl. handle.net/10449/17669.

[25] Pedri U., Pertoll G. (2012). Die Auswirkungen unterschiedlicher Standorte auf Traubenund Weinqualität bei der Sorte 'Sauvignon Blanc'. Mitteilungen Klosterneuburg 62 (4), 123-142.

[26] Köppen W. (1918). Klassification der Klimate nach Temperatur, Niederschlag und Jahreslauf. Dr. A. Petermann's Mitteilungen aus Justus Perthes' Geographischer Anstalt 64, 193-203.

[27] Thalheimer M. (2006). Kartierung der landwirtschaftlich genutzten Böden des Überetsch in Südtirol. Laimburg Journal 3 (1), 135 177

[28] Huglin P. (1978). Nouveau mode d'évaluation des possibilités héliothermiques d'un milieu viticole. Comptes Rendus de I'Academie d'Agriculture de France 64, 1117-1126.

[29] Maaß U., Schwab A. (2012). Temperaturentwicklung Laimburg im Vergleich mit anderen europäischen Weinbaustandorten. Obstbau Weinbau Fachblatt des Südtiroler Beratungsringes 49 (2), 72-75.

[30] Seguin B., Garcia de Cortazar I. (2005). Climate Warming: Consequences for Viticolture and the Notion of 'Terroirs' in Europe. Acta Horticulturae International Society for Horticultural Science (ISHS) 689, 61-70, DOI: 10.17660/ActaHortic.2005.689.3.

[31] Petgen M. (2007). Reaktion der Reben auf den Klimawandel. Schweizerische Zeitschrift für Obst- und Weinbau 143 (9), 6-9.

[32] Maaß U., Schwab A. (2011). Klimawandel und Sortenwahl. Der Huglin-Index und der Wärmeanspruch von Rebsorten. Das Deutsche Weinmagazin (10), 29-31.

[33] Lorenz D. H., Eichhorn K. W., Bleihofer H. Klose R., Meier U., Weber E. (1994). Phänologische Entwicklungsstadien der Weinrebe (Vitis vinifera L. ssp. Vinifera). Codierung und Beschreibung nach der erweiterten $\mathrm{BBCH}$ Skala. Wein-Wissenschaft 49 (2), 66-70.

[34] Weiss J., Willisch E., Knorr D., Schaller A. (1972). Ergebnisse von Untersuchungen bezüglich der differenzierten Wirkung einer sensorischen bewertenden Prüfmethode gegenüber einer sensorischen RangordnungsPrüfmethode am Beispiel von Apfelsaft und Birnennektar. Confructa 17 (4/5), 237-250.

[35] Kobler A. (1996). La valutazione sensoriale dei vini ed il controllo degli assaggiatori mediante l'uso di schede di analisi sensoriale non strutturate. Rivista di Viticoltura e di Enologia 49 (4), 3-18. Retrieved April 1, 2016 from http://moosmandl.macbay.net//publikationen/conegliano degustazione 1996.pdf. 


\section{ANHANG 1: ABBILDUNGEN}

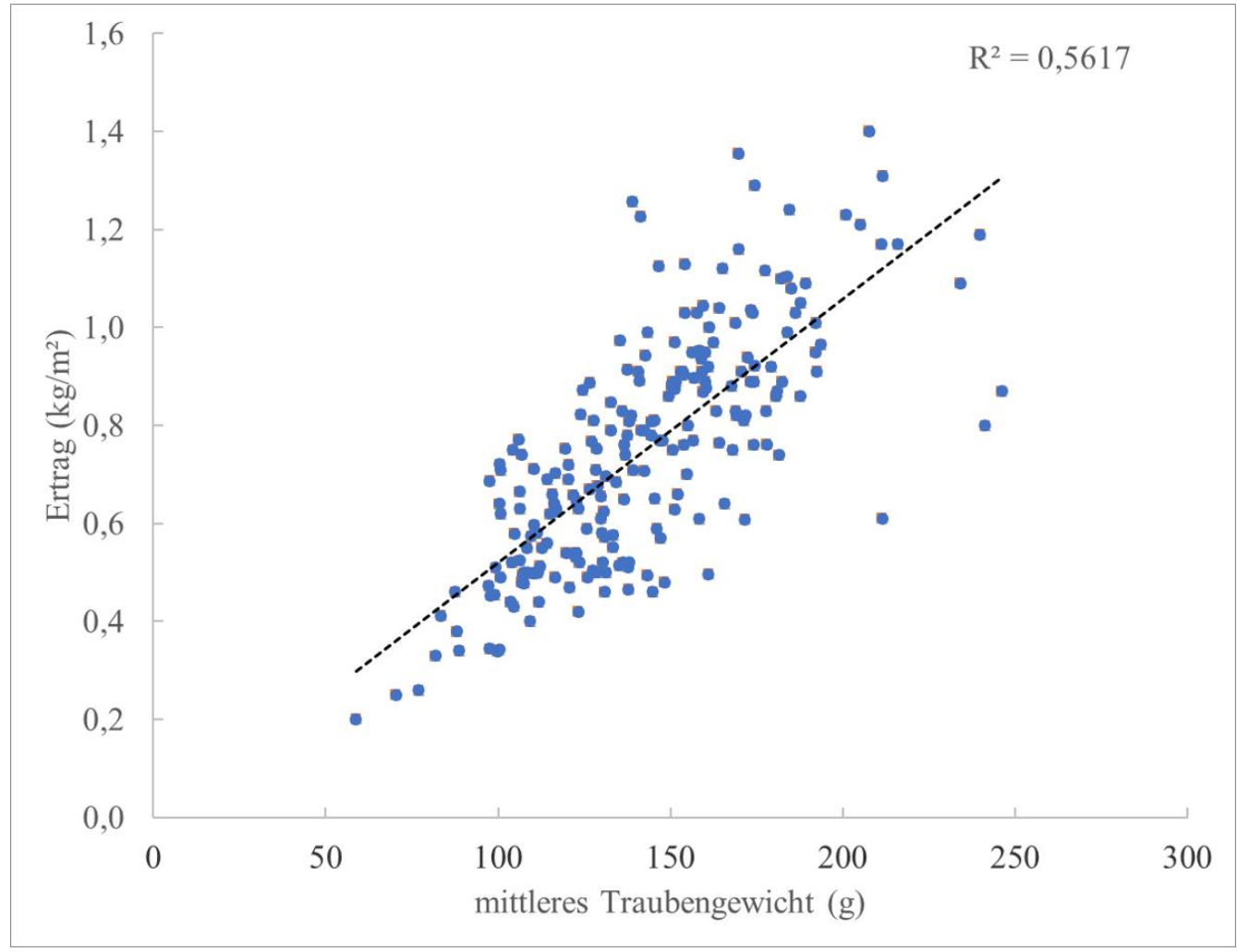

Abb. 1: Korrelationsdiagramm zwischen mittlerem Traubengewicht und Ertrag.

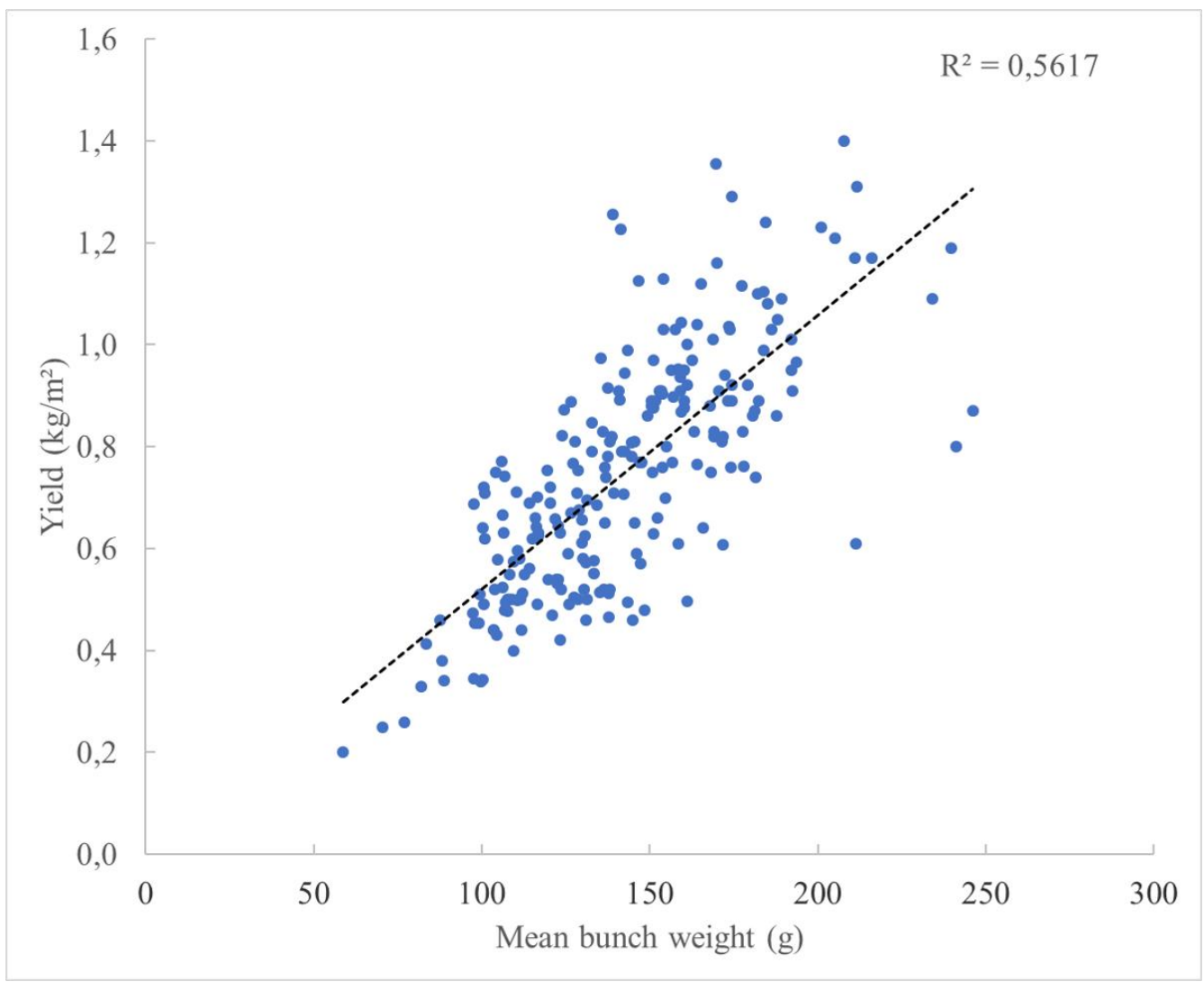

Fig. 1: Correlation diagram of mean berry weight and yield. 


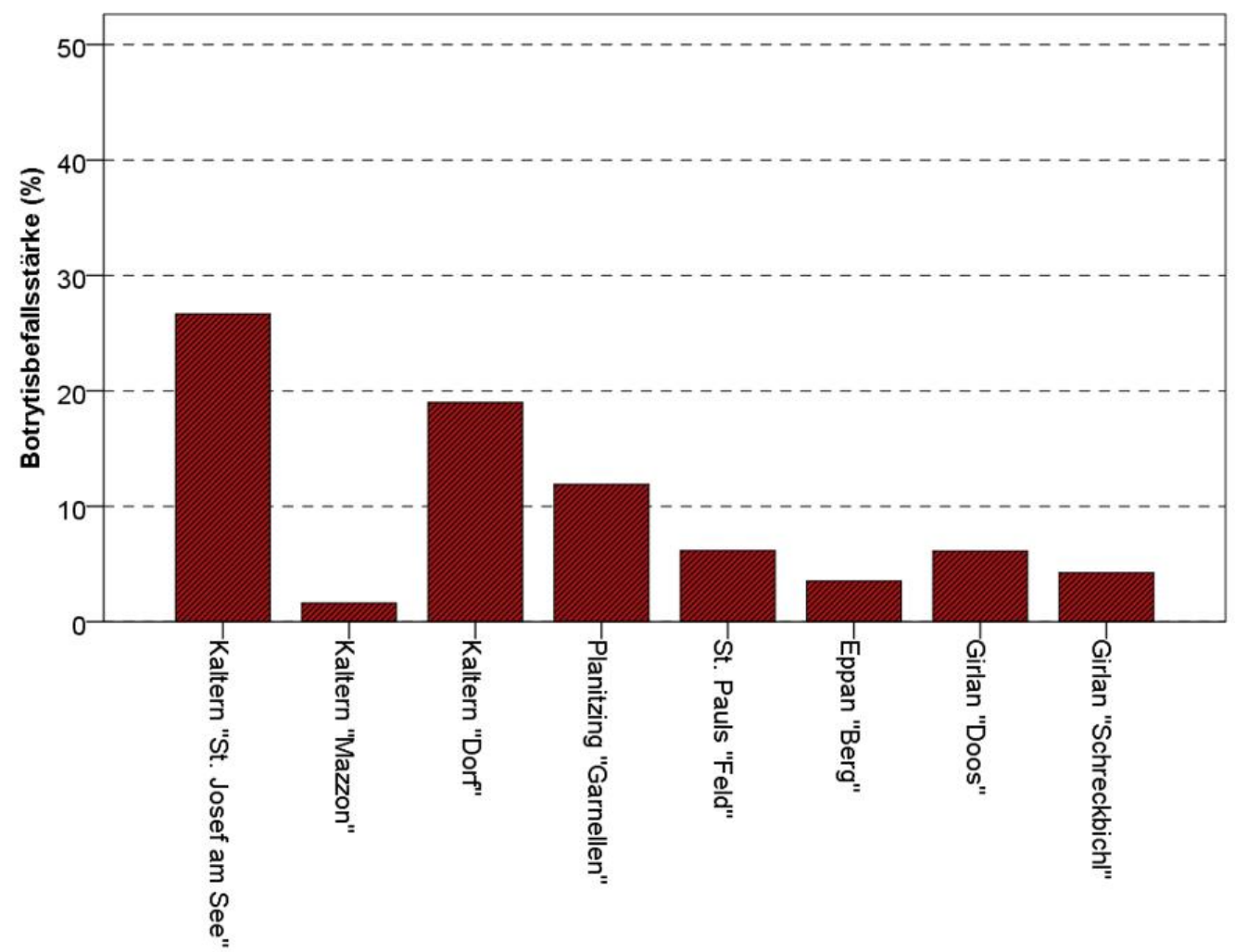

Abb. 2: Balkendiagramm der mittleren Botrytisbefallsstärke in Prozent.

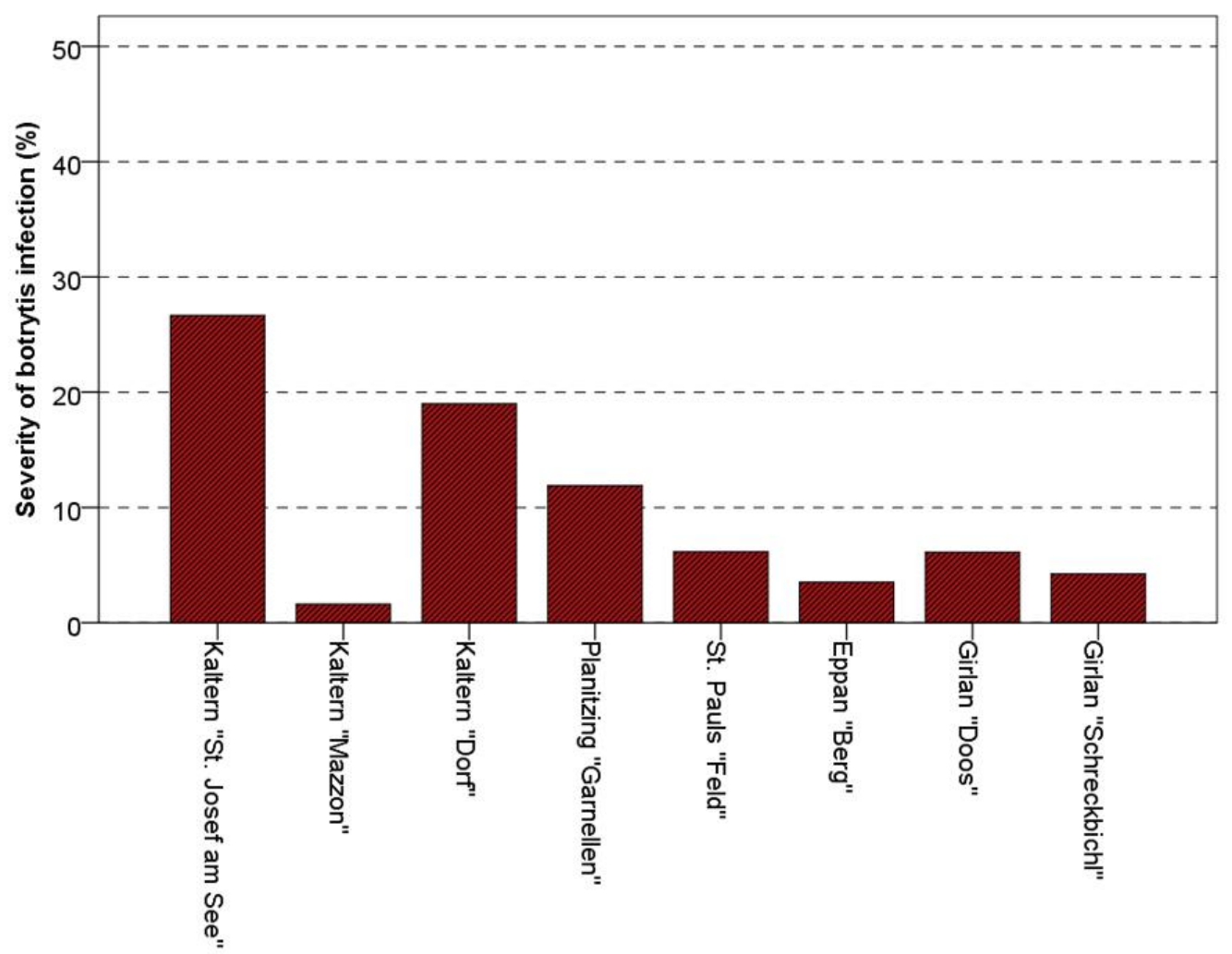

Fig. 2: Bar chart of mean severity of botrytis infection in percent. 


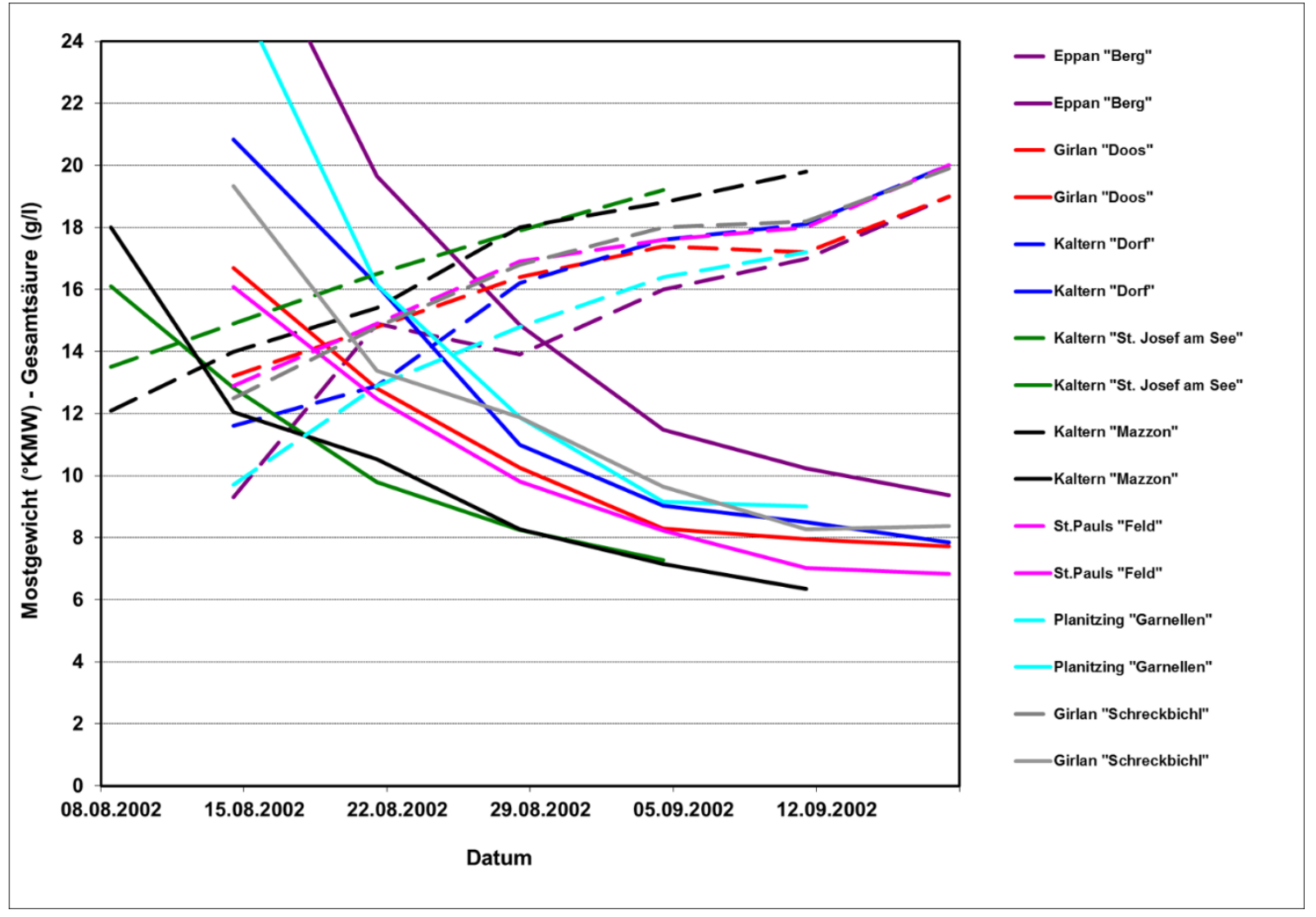

Abb. 3: Reifeverlauf im Jahr 2002 mit Mostgewicht (Klosterneuburger Mostwaage - gestrichelte Linien) und Gesamtsäure (g/l - durchgehende Linie) bei Blauburgunder im Weinanbaugebiet Überetsch.

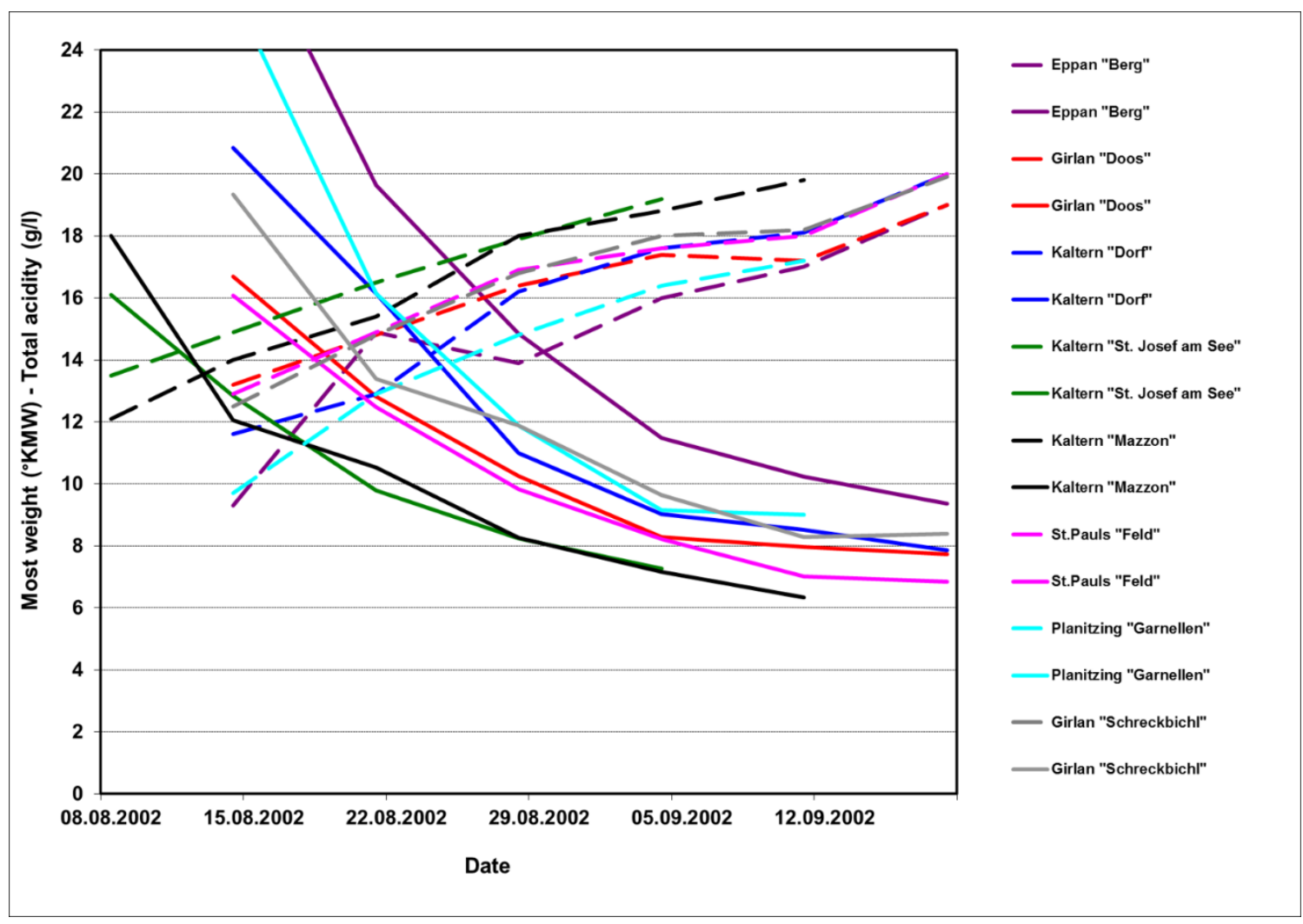

Fig. 3: Maturation in the year 2002, with must weight (Klosterneuburger must scale - dashed line) and total acid (g/l - solid line) for Pinot Noir in the wine-growing area of Überetsch. 


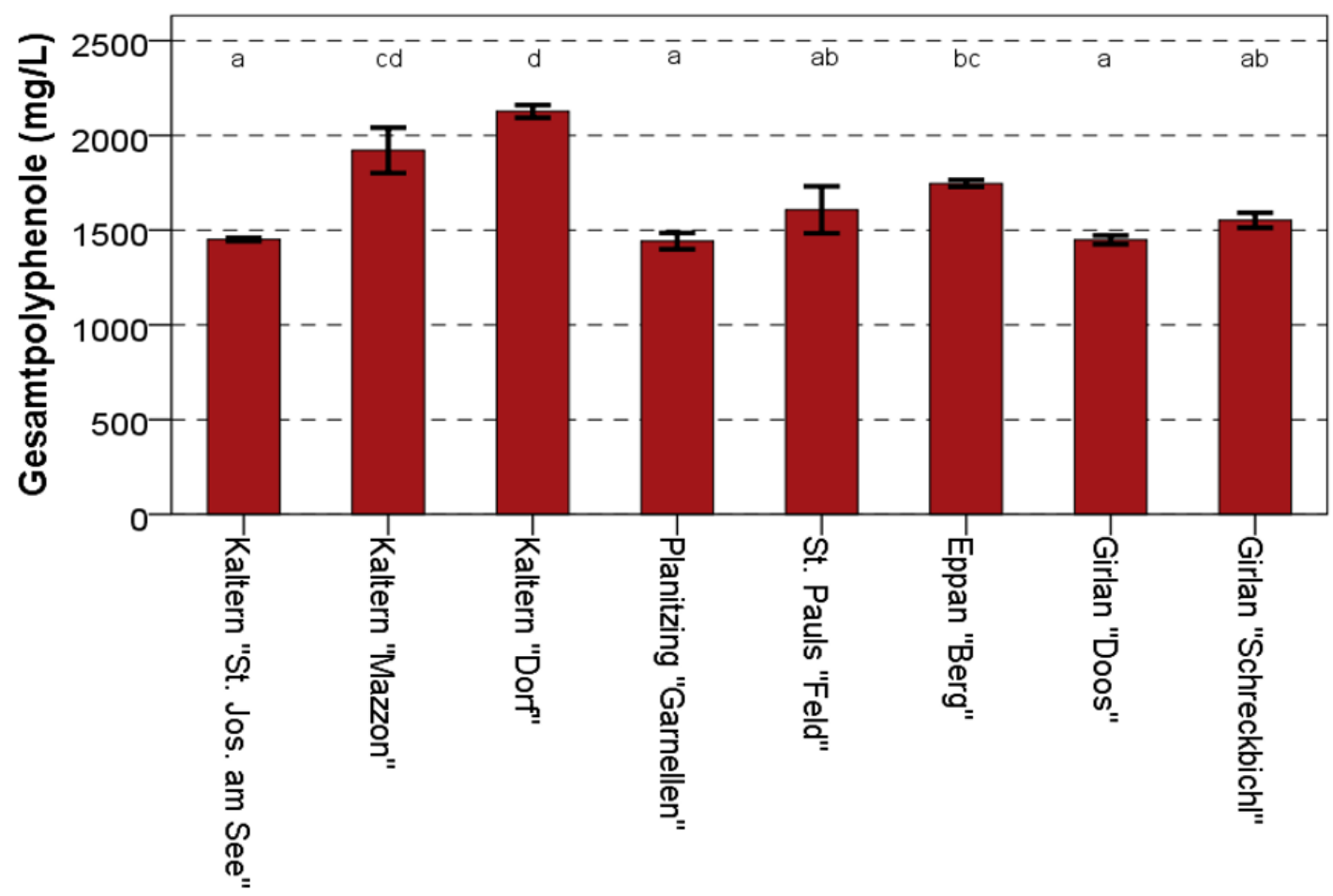

Abb. 4: Gesamtpolyphenole nach Folin-Ciocalteau im Mittel der Jahre mit Standardabweichung.

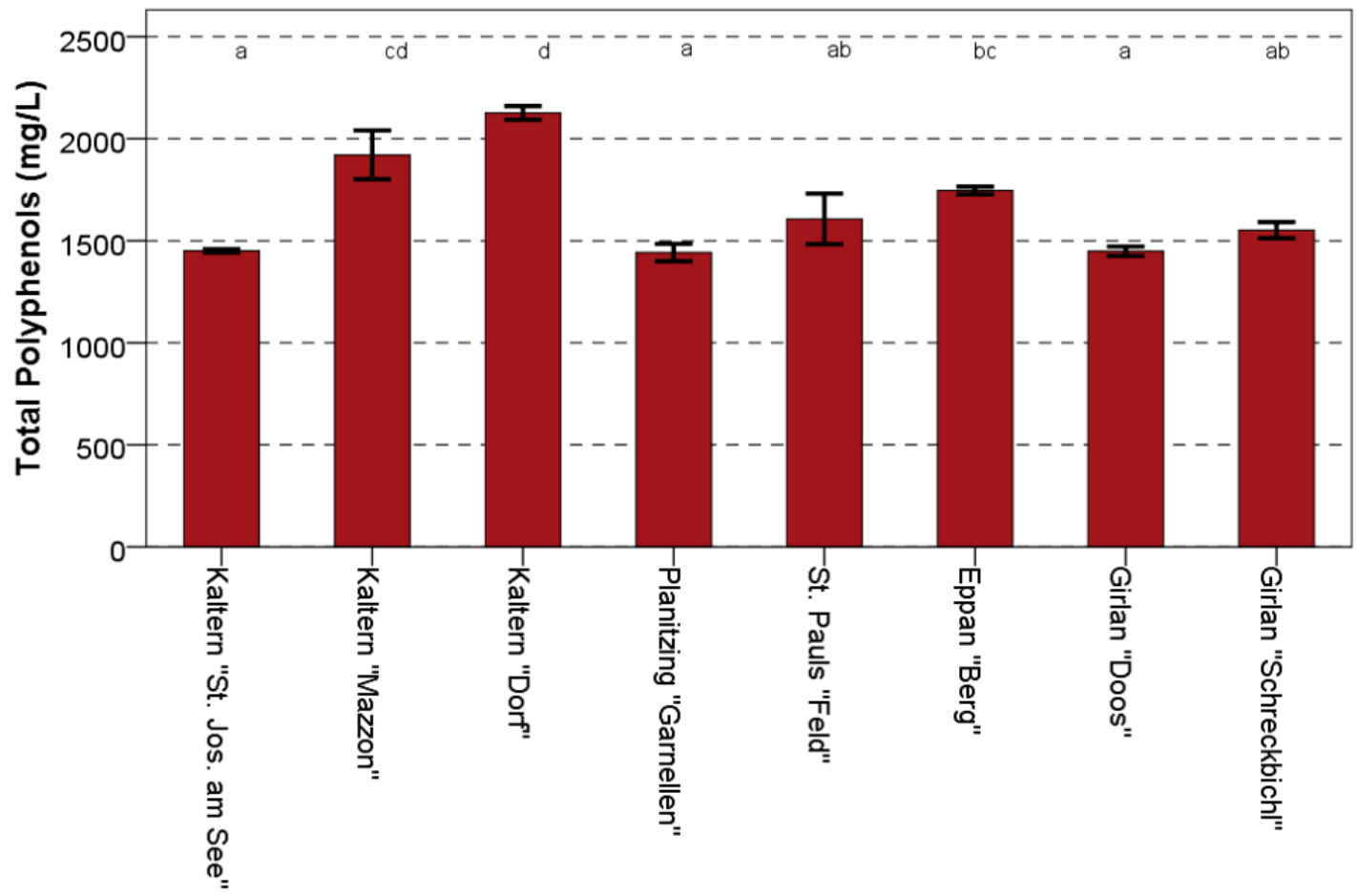

Fig. 4: Total polyphenols according to Folin-Ciocalteau, mean values over course of years, with standard deviation. 


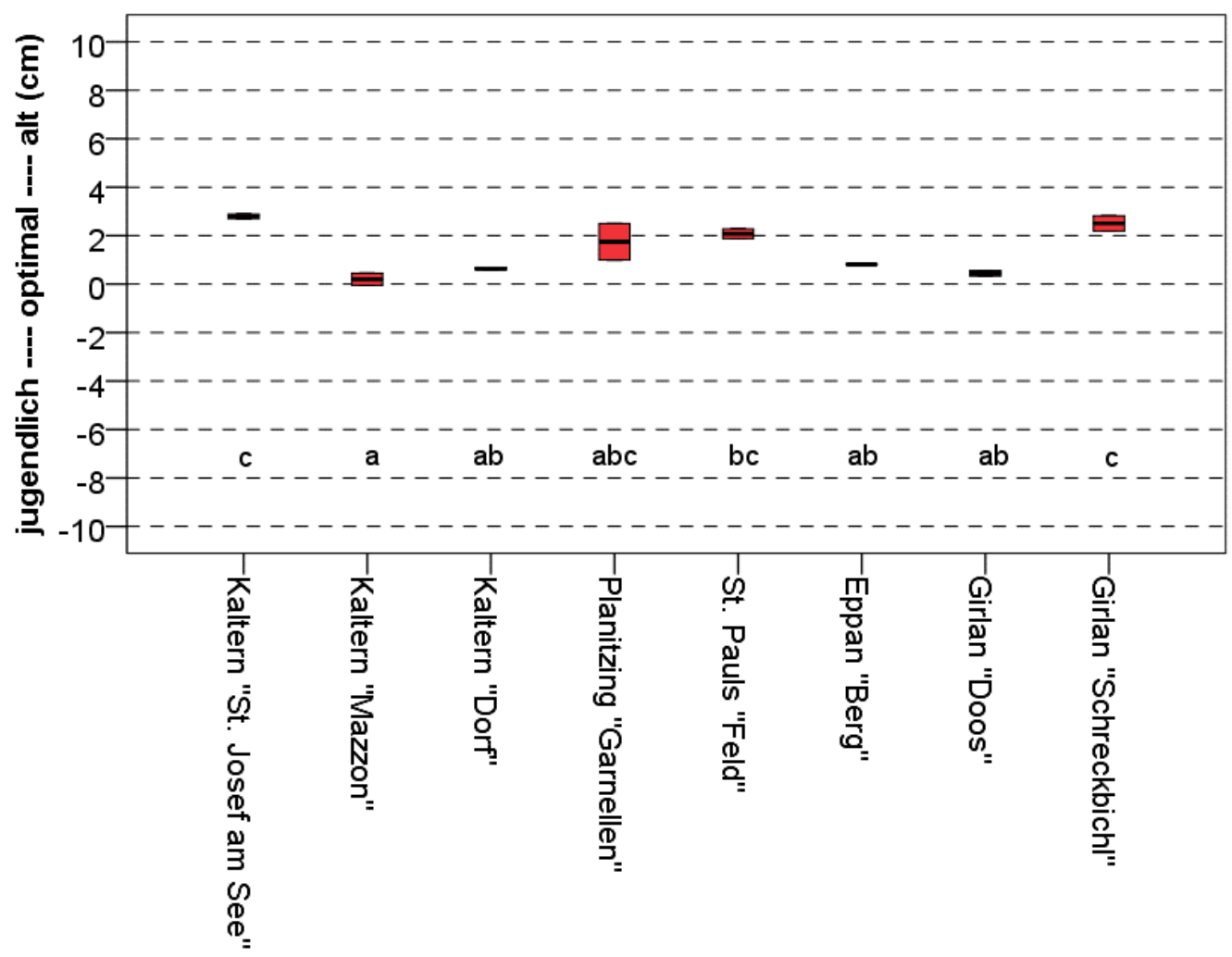

Abb. 5: Beurteilung des Entwicklungszustandes der Weine aus den geprüften Standorten im Schnitt der Jahre von 1996 bis 1999.

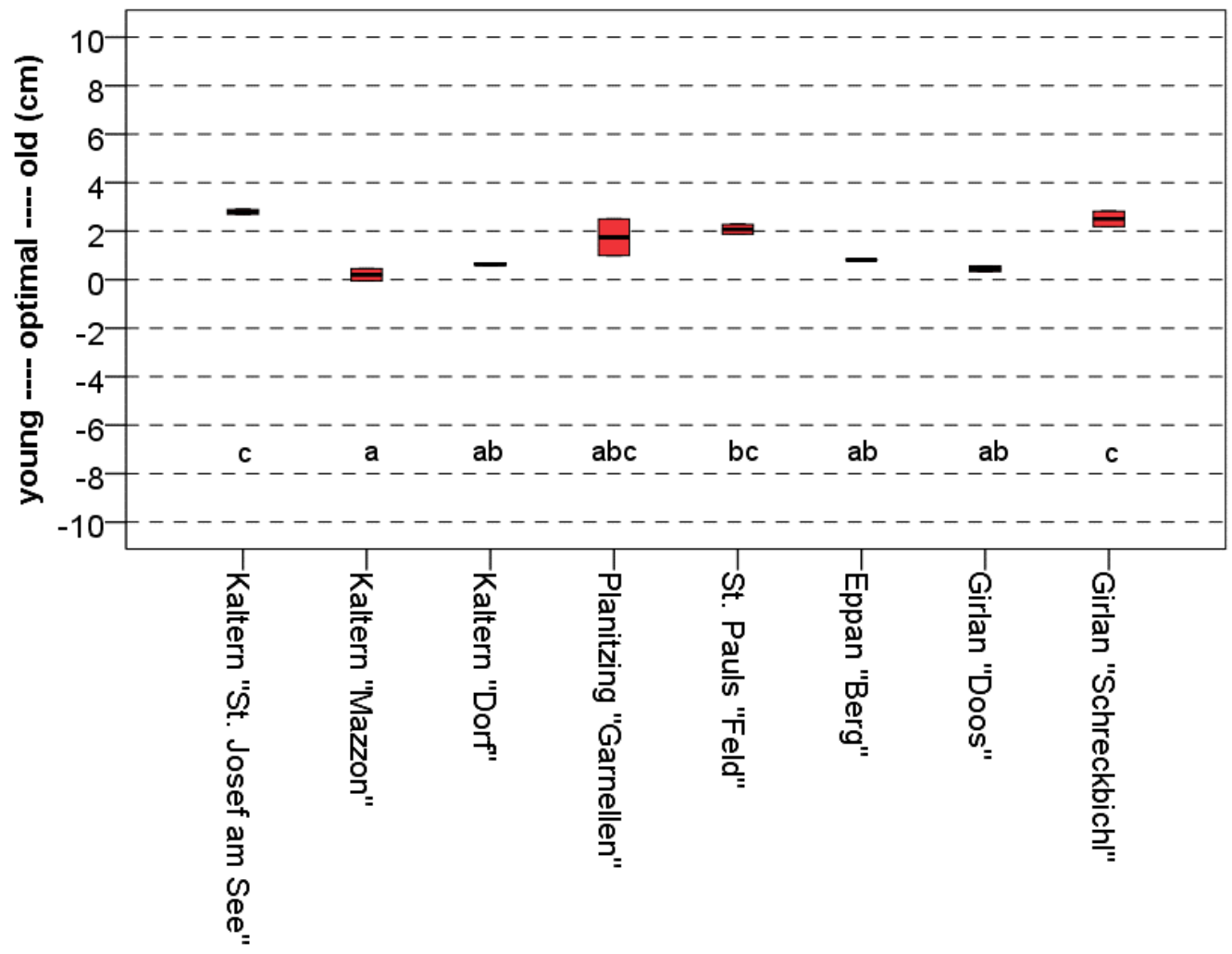

Fig. 5: Evaluation of the developmental condition of the wines from the experimental sites, average values for 1996 to 1999. 


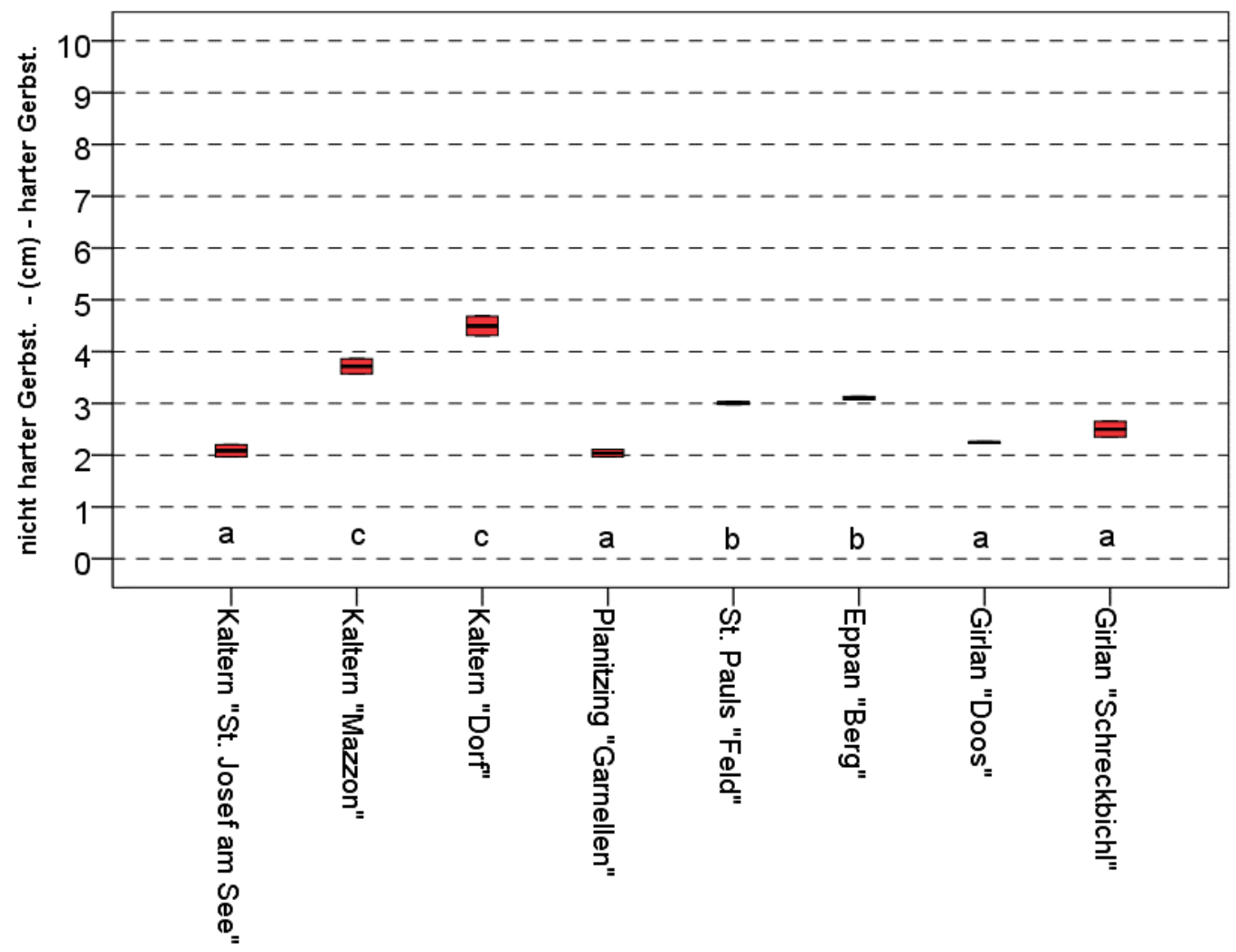

Abb. 6: Beurteilung der sensorisch wahrgenommenen "Härte" des Gerbstoffes der Weine aus den geprüften Standorten im Schnitt der Jahre von 1996 bis 1999.

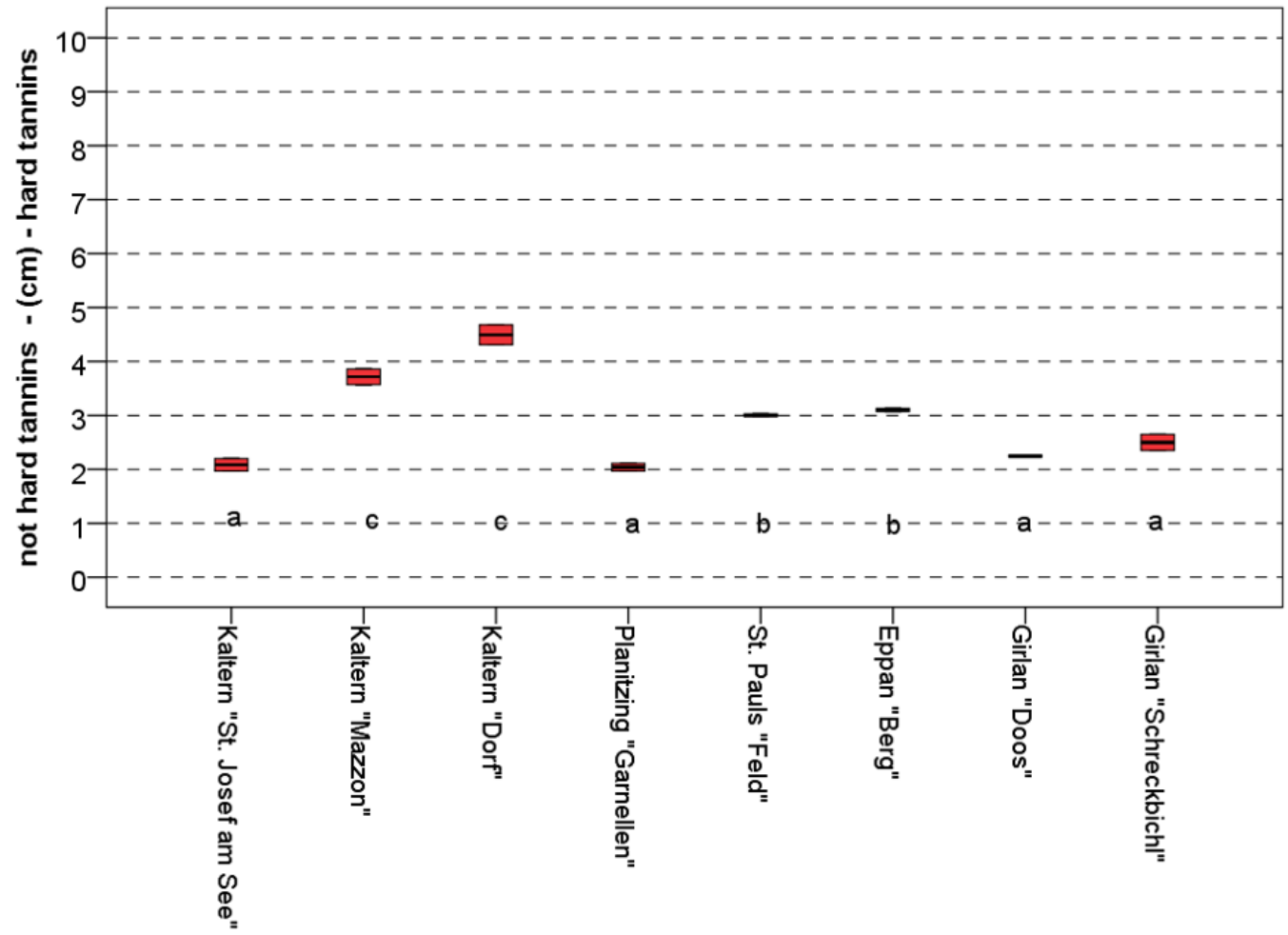

Fig. 6: Evaluation of the sensory perception of "Hardness" of the tannins of the wines from the experimental sites, average values for 1996 to 1999. 


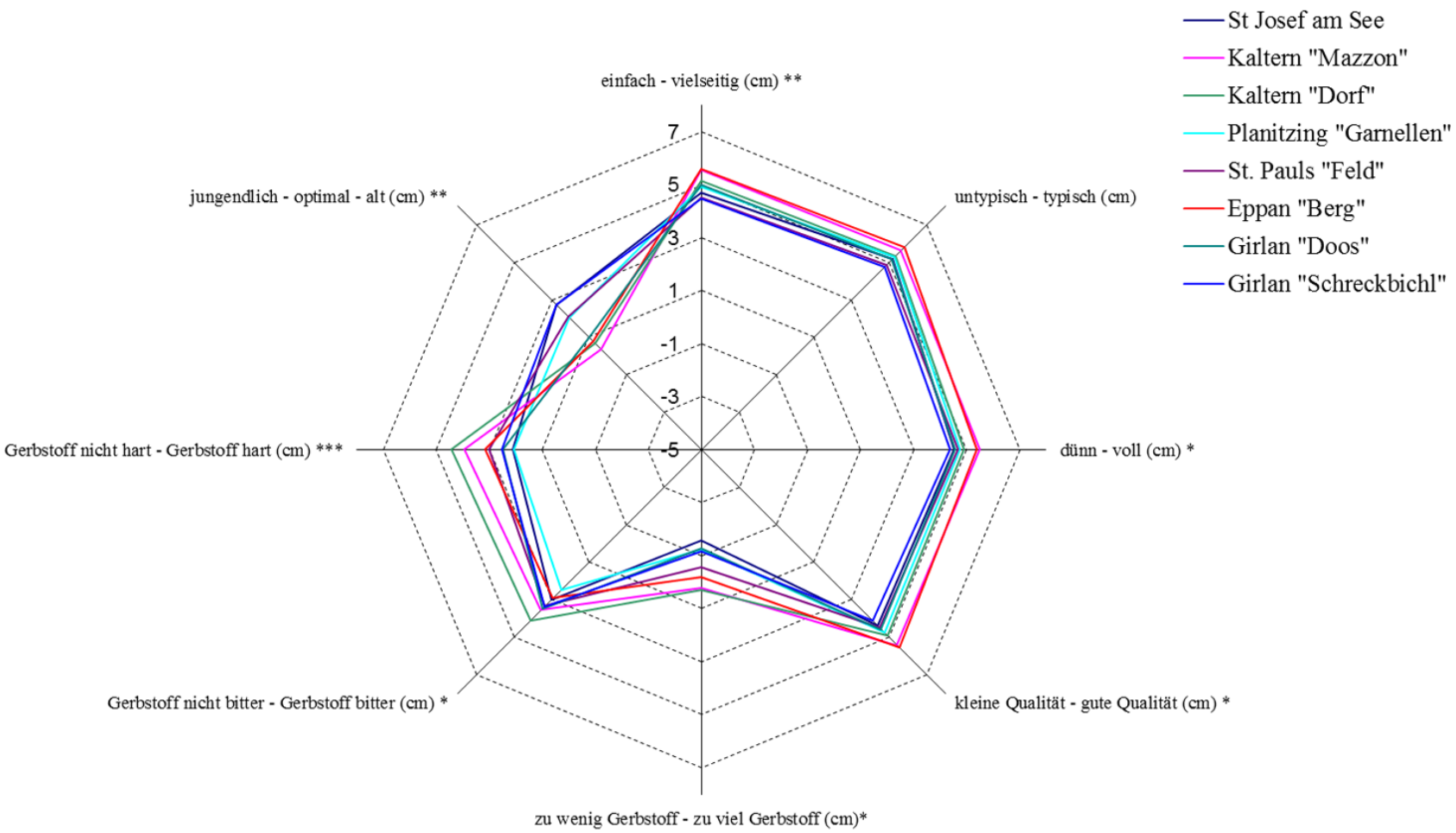

Abb. 7: Sensorisches Profil im Schnitt der Jahre.

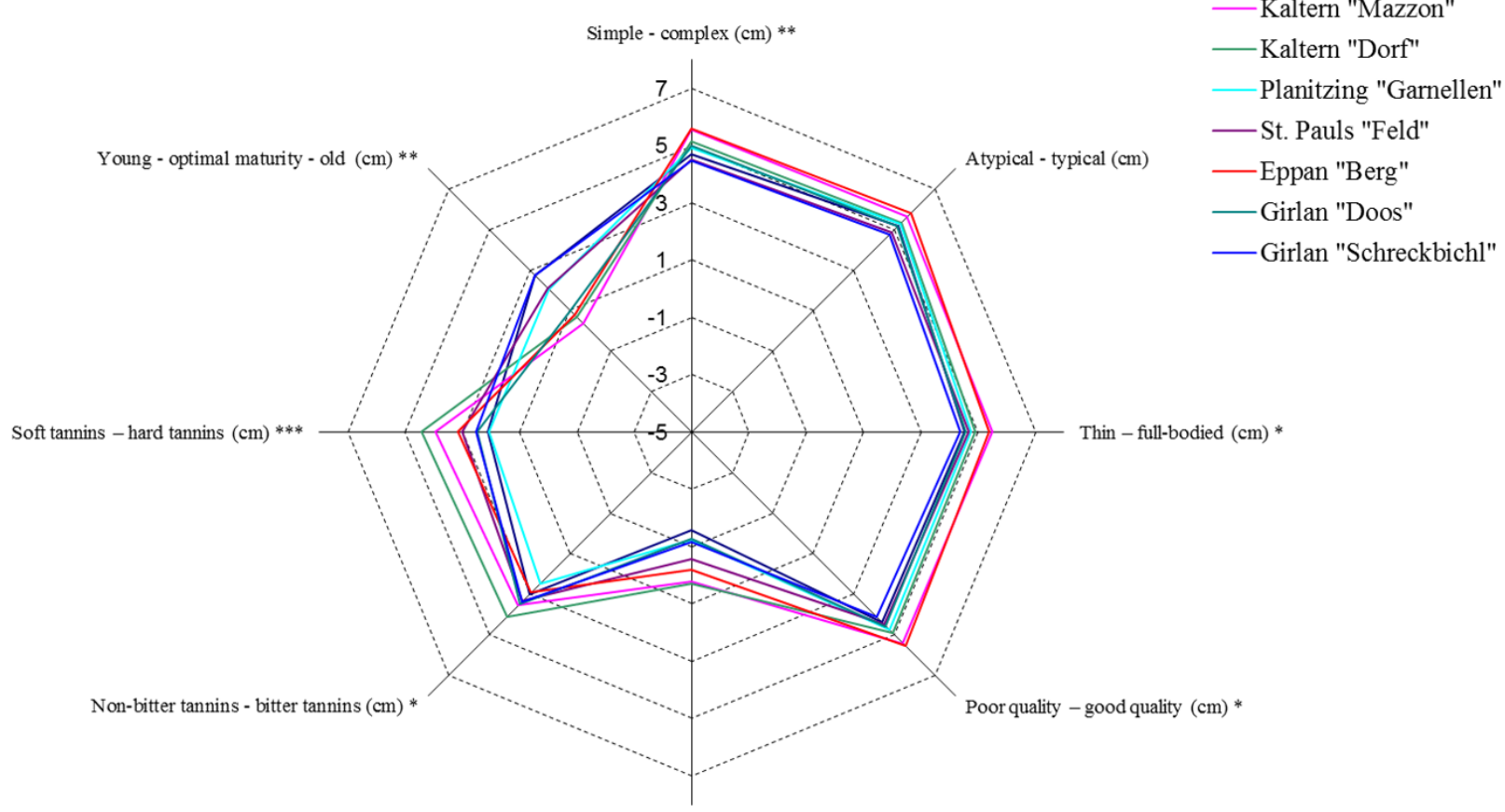

Too little tannins - too much tannins $(\mathrm{cm})$ *

Fig. 7: Sensory profile, yearly average. 


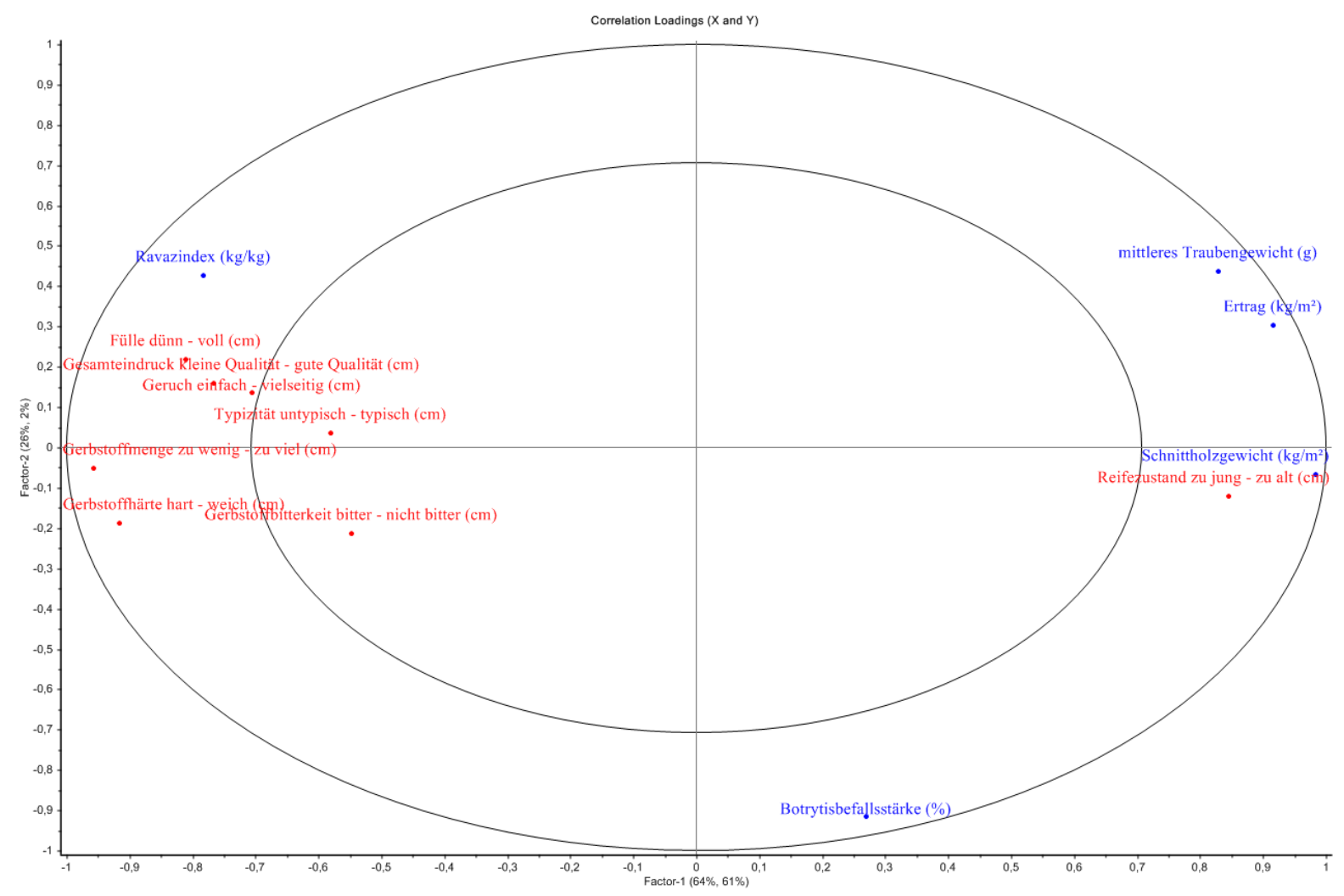

Abb. 8: PLS- (Partial Least Square) Kalibration zwischen weinbaulichen Erhebungen und den sensorischen Eigenschaften der Blauburgunder-Weine.

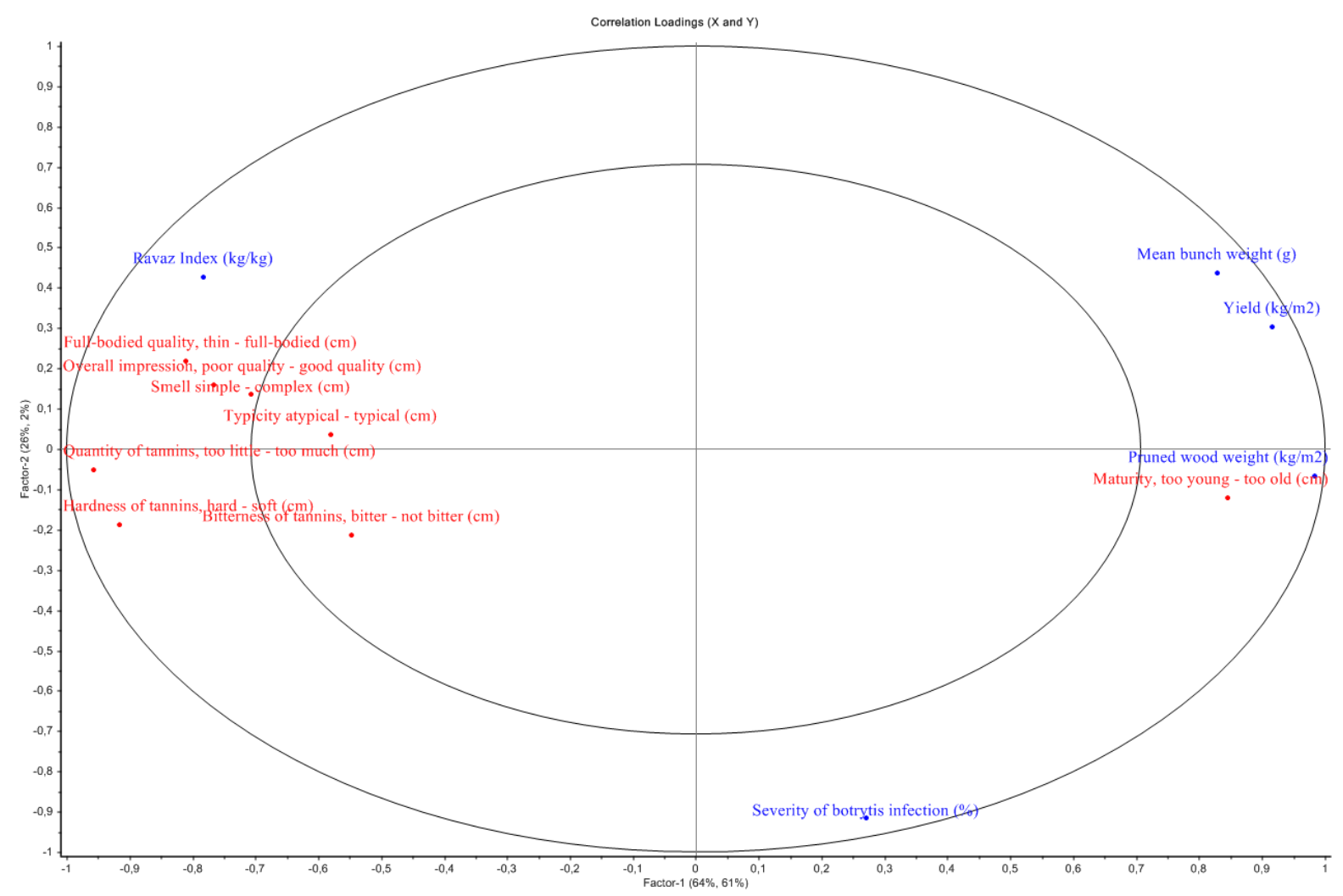

Fig. 8: PLS- (Partial Least Square) Calibration between oenological surveys and the sensory characteristics of Pinot Noir wines. 


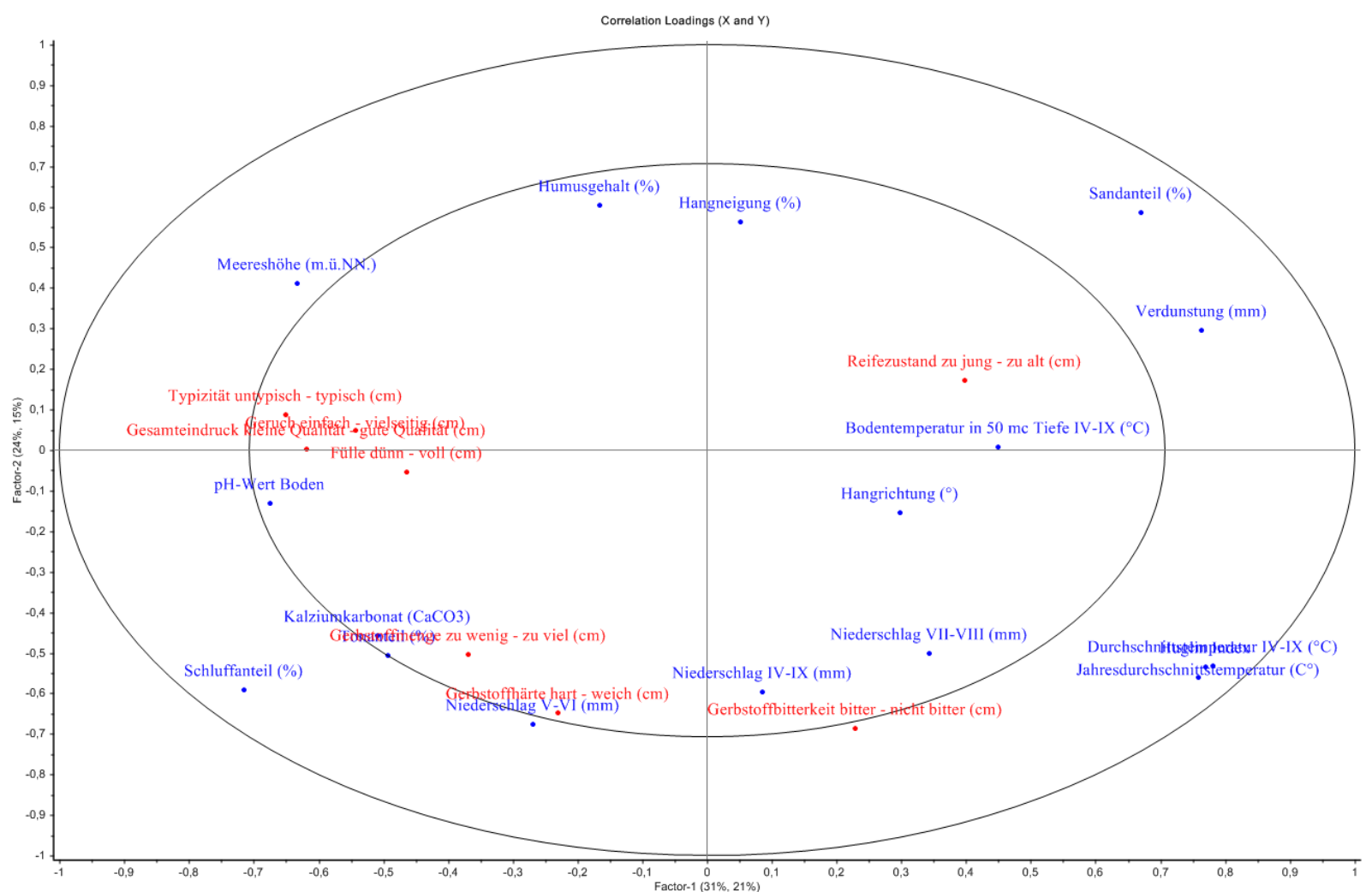

Abb. 9: PLS- (Partial Least Square) Kalibration zwischen Standortkennzahlen, Witterungsdaten und den sensorischen Eigenschaften der Blauburgunder-Weine.

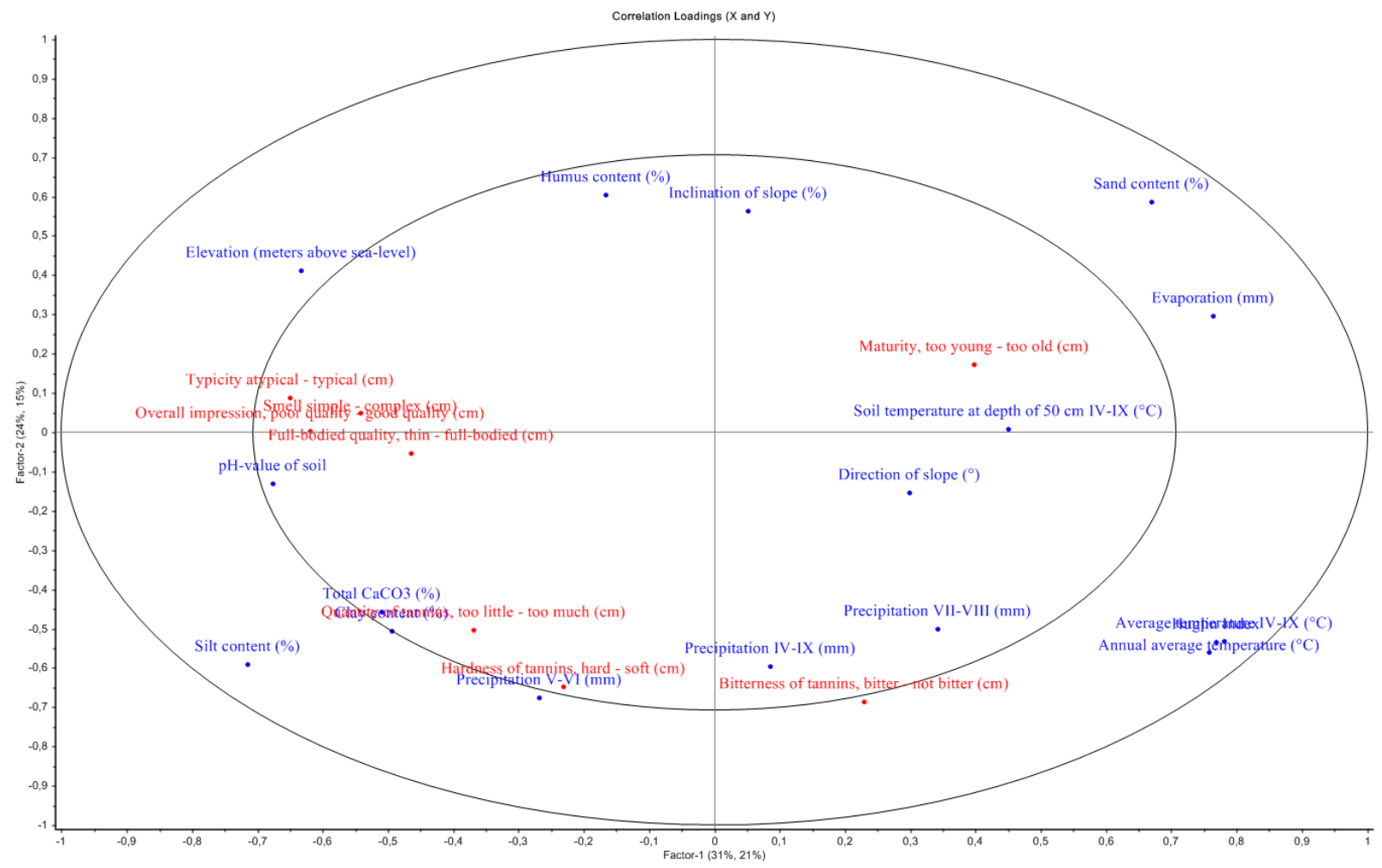

Fig. 9: PLS- (Partial Least Square) Calibration between site characteristics, weather data, and sensory characteristics of Pinot Noir wines. 


\section{ANHANG 2: TABELLEN}

Tab. 1: Topografische und geologische Beschreibung der geprüften Weinbaulagen // Topographical and geological description of investigated winegrowing locations.

\begin{tabular}{|c|c|c|c|c|c|c|c|c|c|c|}
\hline $\begin{array}{l}\text { Versuchslage } \\
\text { Experimental } \\
\text { site }\end{array}$ & $\begin{array}{l}\text { Meeres- } \\
\text { höhe } \\
\text { Elevation } \\
\text { (m) }\end{array}$ & $\begin{array}{c}\text { Hang- } \\
\text { neigung } \\
\text { Inclination } \\
\text { (Grad) }\end{array}$ & $\begin{array}{c}\text { Exposition } \\
\text { Exposition } \\
\text { (Grad) }\end{array}$ & $\begin{array}{l}\text { pH-Boden } \\
\text { pH of soil }\end{array}$ & $\begin{array}{l}\text { Ausgangsgestein } \\
\text { Parent rock } \\
\text { [Bodenart } \\
\text { Soil type*] }\end{array}$ & $\begin{array}{c}\text { Humus } \\
\text { Humus } \\
\text { (\%) }\end{array}$ & $\begin{array}{l}\text { Ton } \\
\text { Clay }\end{array}$ & $\begin{array}{l}\text { Schluff } \\
\text { Silt }\end{array}$ & $\begin{array}{l}\text { Sand } \\
\text { Sand }\end{array}$ & $\begin{array}{c}\text { Gesamt } \\
\text { CaCO3 } \\
\text { Total } \\
\text { CaCO3 } \\
\text { (\%) }\end{array}$ \\
\hline $\begin{array}{l}\text { Eppan } \\
\text { "Berg" }\end{array}$ & 572 & 8 & 105 & 7.15 & $\begin{array}{c}\text { Kalkdeckschutt } \\
\text { Calcareous sur- } \\
\text { face rubble } \\
{[\mathrm{sL}] /[\mathrm{sC}]}\end{array}$ & 4.5 & 18.8 & 41.6 & 39.6 & 36.2 \\
\hline $\begin{array}{l}\text { Girlan } \\
\text { "Doos" }\end{array}$ & 420 & 5 & 330 & 6.10 & $\begin{array}{l}\text { Moräne } \\
\text { Moraine } \\
{[\mathrm{sL}] /[\mathrm{sC}]}\end{array}$ & 4.1 & 8.0 & 26.3 & 65.7 & 0.0 \\
\hline $\begin{array}{l}\text { Kaltern } \\
\text { "Dorf" }\end{array}$ & 458 & 4 & 140 & 7.15 & $\begin{array}{c}\text { Kalkdeckschutt } \\
\text { Calcareous sur- } \\
\text { face rubble } \\
{[\mathrm{sL}] /[\mathrm{sC}]}\end{array}$ & 3.3 & 20.3 & 50.7 & 29.0 & 51.0 \\
\hline $\begin{array}{l}\text { St. Josef } \\
\text { am See }\end{array}$ & 247 & 5 & 80 & 7.25 & $\begin{array}{c}\text { Kalkdeckschutt } \\
\text { Calcareous sur- } \\
\text { face rubble } \\
{[\mathrm{sL}] /[\mathrm{sC}]}\end{array}$ & 3.0 & 21.3 & 42.0 & 36.7 & 55.3 \\
\hline $\begin{array}{l}\text { Kaltern } \\
\text { "Mazzon" }\end{array}$ & 383 & 5 & 160 & 6.50 & $\begin{array}{l}\text { Moräne } \\
\text { Moraine } \\
{[\mathrm{sL}] /[\mathrm{sC}]}\end{array}$ & 3.0 & 10.8 & 26.0 & 63.2 & 0.0 \\
\hline $\begin{array}{l}\text { St. Pauls } \\
\text { "Feld" }\end{array}$ & 375 & 2.5 & 350 & 6.15 & $\begin{array}{l}\text { Seenablagerung } \\
\text { Lacustrine de- } \\
\text { posits } \\
{[\mathrm{IS}] /[\mathrm{cS}]}\end{array}$ & 3.0 & 13.0 & 34.0 & 53.0 & 0.0 \\
\hline $\begin{array}{l}\text { Planitzing } \\
\text { "Garnellen" }\end{array}$ & 482 & 4 & 105 & 7.15 & $\begin{array}{c}\text { Kalkdeckschutt } \\
\text { Calcareous sur- } \\
\text { face rubble } \\
{[\mathrm{sL}] /[\mathrm{sC}]}\end{array}$ & 3.2 & 11.3 & 34.3 & 54.4 & 0.0 \\
\hline $\begin{array}{c}\text { Girlan } \\
\text { "Schreckbichl" }\end{array}$ & 468 & 10 & 110 & 6.30 & $\begin{array}{c}\text { Moräne } \\
\text { Moraine } \\
\text { [hlS]/[hcS] }\end{array}$ & 4.5 & 11.8 & 18.0 & 70.2 & 0.0 \\
\hline
\end{tabular}


Tab. 2: Klimadaten der Versuchsstandorte im Südtiroler Überetsch (Mittelwerte 1996-2002) // Climatic data for experimental sites in the Überetsch area of South Tyrol (means values for 1996-2002).

\begin{tabular}{|c|c|c|c|c|c|c|c|}
\hline $\begin{array}{l}\text { Versuchsanlage } \\
\text { Experimental site }\end{array}$ & $\begin{array}{l}\text { Lufttemperatur } \\
\text { Air tempera- } \\
\text { ture } \\
\\
2 \mathrm{~m} \\
\left({ }^{\circ} \mathrm{C}\right)\end{array}$ & $\begin{array}{l}\text { Bodentempe- } \\
\text { ratur } \\
\text { Soil tempera- } \\
\text { ture } \\
-50 \mathrm{~cm} \\
\left({ }^{\circ} \mathrm{C}\right)\end{array}$ & $\begin{array}{l}\text { Luftfeuchte } \\
\text { Air humidity }\end{array}$ & $\begin{array}{l}\text { Niederschlag } \\
\text { Precipitation }\end{array}$ & $\begin{array}{l}\text { Niederschlag } \\
\text { Precipitation } \\
\text { April-September } \\
\text { (mm) }\end{array}$ & $\begin{array}{l}\text { Windge- } \\
\text { schwindigkeit } \\
\text { Wind speed } \\
(\mathrm{m} / \mathrm{s})\end{array}$ & $\begin{array}{l}\text { Wind- } \\
\text { richtung } \\
\text { Wind } \\
\text { direction }\end{array}$ \\
\hline $\begin{array}{l}\text { Eppan } \\
\text { "Berg" }\end{array}$ & 10.7 & 11.3 & 71.3 & 954.1 & 594.2 & 0.95 & $\begin{array}{l}\text { SSW } \\
\text { SSW }\end{array}$ \\
\hline $\begin{array}{l}\text { Girlan } \\
\text { "Doos" }\end{array}$ & 11.9 & 11.0 & 69.5 & 858.5 & 524.5 & 0.97 & $\begin{array}{l}\text { SSO } \\
\text { SSE }\end{array}$ \\
\hline $\begin{array}{l}\text { Kaltern } \\
\text { "Dorf" }\end{array}$ & 12.1 & 11.2 & 71.1 & 1085.8 & 662.0 & 0.75 & $\begin{array}{l}\text { SSW } \\
\text { SSW }\end{array}$ \\
\hline $\begin{array}{c}\text { Kaltern } \\
\text { "St. Josef am See" }\end{array}$ & 12.3 & 12.4 & 72.1 & 1051.0 & 644.0 & 1.23 & $\begin{array}{l}\text { SSW } \\
\text { SSW }\end{array}$ \\
\hline $\begin{array}{l}\text { Kaltern } \\
\text { "Mazzon" }\end{array}$ & 12.3 & 12.0 & 72.2 & 1003.3 & 606.6 & 1.44 & $\begin{array}{l}\text { SSO } \\
\text { SSE }\end{array}$ \\
\hline $\begin{array}{l}\text { St. Pauls } \\
\text { "Feld" }\end{array}$ & 11.9 & 10.4 & 68.1 & 907.5 & 557.5 & 0.91 & $\begin{array}{l}\text { SSO } \\
\text { SSE }\end{array}$ \\
\hline $\begin{array}{l}\text { Planitzing } \\
\text { "Garnellen" }\end{array}$ & 11.2 & 11.3 & 71.2 & 997.4 & 612.5 & 1.20 & $\begin{array}{l}\text { SSW } \\
\text { SSW }\end{array}$ \\
\hline $\begin{array}{c}\text { Girlan } \\
\text { "Schreckbichl" }\end{array}$ & 11.8 & 11.8 & 69.3 & 979.7 & 614.1 & 1.79 & $\begin{array}{l}\text { SSW } \\
\text { SSW }\end{array}$ \\
\hline
\end{tabular}

Tab. 3: Huglin-Index der acht Versuchsstandorte im Überetsch vom Sorten-Lagen-Projekt // Huglin Index of the eight experimental sites in the Überetsch area of Variety/Site Project.

\begin{tabular}{|c|c|c|c|c|c|c|c|c|}
\hline $\begin{array}{l}\text { Jahr } \\
\text { Year }\end{array}$ & $\begin{array}{l}\text { St. Pauls } \\
\text { "Feld" }\end{array}$ & $\begin{array}{l}\text { Eppan } \\
\text { "Berg" }\end{array}$ & $\begin{array}{c}\text { Girlan } \\
\text { "Schreckbichl" }\end{array}$ & $\begin{array}{l}\text { Planitzing } \\
\text { "Garnellen" }\end{array}$ & $\begin{array}{l}\text { Kaltern } \\
\text { "Dorf" }\end{array}$ & $\begin{array}{l}\text { Kaltern } \\
\text { "Mazzon" }\end{array}$ & $\begin{array}{c}\text { Kaltern } \\
\text { "St. Josef am } \\
\text { See" }\end{array}$ & $\begin{array}{l}\text { Girlan } \\
\text { "Doos" }\end{array}$ \\
\hline 1996 & 2168 & 1833 & 2064 & 1906 & 2029 & 2077 & 2299 & 2107 \\
\hline 1997 & 2218 & 1889 & 2140 & 2006 & 2150 & 2327 & 2396 & 2186 \\
\hline 1998 & 2280 & 1886 & 2227 & 2080 & 2402 & 2424 & 2505 & 2240 \\
\hline 1999 & 2269 & 1849 & 2492 & 2009 & 2344 & 2245 & 2454 & 2204 \\
\hline 2000 & 2323 & 1964 & 2112 & 2104 & 2344 & 2278 & 2393 & 2311 \\
\hline 2001 & 2203 & 1790 & 1911 & 1945 & 2150 & 2153 & 2130 & 2010 \\
\hline 2002 & n.v. & n.v. & 1953 & 1918 & n.v. & 2006 & 2126 & 2124 \\
\hline $\begin{array}{l}\text { ittelwert } \\
\text { an value }\end{array}$ & 2244 & 1869 & 2128 & 1995 & 2237 & 2216 & 2329 & 2169 \\
\hline
\end{tabular}


Tab. 4: Ergebnisse der Varianzanalyse weinbaulicher Messwerte (nur Varianten mit unterschiedlichen Buchstaben unterscheiden sich signifikant voneinander, Einfakt. Anova, Tukey-B, Signifikanzniveau 0.05) // Results of variance analysis of oenological measurement values (only variants with different letters differ significantly from each other, Einfakt. Anova, Tukey-B, Significance level 0.05).

\begin{tabular}{|c|c|c|c|c|c|c|c|c|c|}
\hline Parameter & & $\begin{array}{l}\text { Kaltern } \\
\text { "St. Josef } \\
\text { am See" }\end{array}$ & $\begin{array}{l}\text { Kaltern } \\
\text { "Mazzon" }\end{array}$ & $\begin{array}{l}\text { Kaltern } \\
\text { "Dorf" }\end{array}$ & $\begin{array}{l}\text { Planitzing } \\
\text { "Garnel- } \\
\text { len" }\end{array}$ & $\begin{array}{l}\text { St. Pauls } \\
\text { "Feld" }\end{array}$ & $\begin{array}{l}\text { Eppan } \\
\text { "Berg" }\end{array}$ & $\begin{array}{l}\text { Girlan } \\
\text { "Doos" }\end{array}$ & $\begin{array}{l}\text { Girlan } \\
\text { "Schreck- } \\
\text { bichl" }\end{array}$ \\
\hline \multicolumn{10}{|l|}{$\begin{array}{l}\text { Weinbau } \\
\text { Wine-growing }\end{array}$} \\
\hline $\begin{array}{l}\text { Ertrag }\left(\mathrm{kg} / \mathrm{m}^{2}\right) \\
\text { Yield }\left(\mathrm{kg} / \mathrm{m}^{2}\right)\end{array}$ & $* * *$ & $0.88 \mathrm{c}$ & $0.70 a b$ & $0.61 \quad a$ & $0.75 \quad b$ & $0.72 \quad b$ & $0.73 \quad b$ & $0.74 \quad b$ & $0.87 \mathrm{c}$ \\
\hline $\begin{array}{l}\text { mittleres Traubenge- } \\
\text { wicht (g) } \\
\text { Mean berry weight }(g)\end{array}$ & $* *$ & $147.7 \mathrm{ab}$ & $142.4 \mathrm{ab}$ & $132.4 \quad a$ & $144.8 \mathrm{ab}$ & $141.2 \mathrm{a}$ & $135.7 \quad a$ & $136.7 \quad a$ & $159.1 \mathrm{~b}$ \\
\hline $\begin{array}{l}\text { Schnittholzgewicht } \\
\text { Pruned wood weight } \\
\left(\mathrm{kg} / \mathrm{m}^{2}\right)\end{array}$ & $* * *$ & $0.28 \mathrm{~d}$ & $0.10 \quad a$ & $0.16 a b$ & $0.23 \mathrm{bcd}$ & $0.17 a b c$ & $0.22 \mathrm{bcd}$ & $0.24 \mathrm{bcd}$ & $0.25 \mathrm{~cd}$ \\
\hline RAVAZ-Index & $* * *$ & $3.2 \mathrm{a}$ & $7.2 b$ & $4.2 \mathrm{a}$ & $3.0 \mathrm{a}$ & $4.8 \quad \mathrm{a}$ & $3.9 \mathrm{a}$ & $3.8 \mathrm{a}$ & $3.8 \mathrm{a}$ \\
\hline $\begin{array}{l}\text { Botrytisbefallsstärke * } \\
\text { (\%) } \\
\text { Severity of botrytis in- } \\
\text { fection * (\%) }\end{array}$ & $* * *$ & $26.7 \mathrm{f}$ & $1.6 \mathrm{a}$ & $19.0 \mathrm{e}$ & $11.9 \mathrm{~d}$ & $5.9 \mathrm{c}$ & $3.5 \mathrm{~b}$ & $6.1 \mathrm{c}$ & $4.3 \mathrm{~b}$ \\
\hline
\end{tabular}

*Jahre 1996 bis 1999 // From 1996 to 1999. 
Tab. 5: Phänologische Stadien von 1996 bis 2002 an den acht Versuchsstandorten im Südtiroler Überetsch.

\begin{tabular}{|c|c|c|c|c|c|c|c|c|c|}
\hline Stadium & & $\begin{array}{l}\text { Kaltern } \\
\text { "St. Josef } \\
\text { am See" }\end{array}$ & $\begin{array}{l}\text { Kaltern } \\
\text { "Mazzon" }\end{array}$ & $\begin{array}{l}\text { Kaltern } \\
\text { "Dorf" }\end{array}$ & $\begin{array}{l}\text { St. Pauls } \\
\text { "Feld" }\end{array}$ & $\begin{array}{l}\text { Planitzing } \\
\text { "Garnel- } \\
\text { len" }\end{array}$ & Eppan "Berg" & $\begin{array}{l}\text { Girlan } \\
\text { "Doos" }\end{array}$ & $\begin{array}{c}\text { Girlan } \\
\text { "Schreck- } \\
\text { bichl" }\end{array}$ \\
\hline Boden $T>=10^{\circ}$ & \multirow{5}{*}{ ஜे } & 10.04.1996 & 12.04.1996 & 20.04.1996 & 23.04.1996 & 18.04.1996 & 21.04.1996 & 20.04.1996 & 19.04.1996 \\
\hline Austrieb (7) & & 16.04.1996 & 18.04.1996 & 19.04.1996 & 19.04.1996 & 20.04.1996 & 21.04.1996 & 19.04.1996 & 19.04.1996 \\
\hline Blühbeginn & & 27.05.1996 & 01.06.1996 & 03.06.1996 & 01.06.1996 & 06.06.1996 & 07.06.1996 & 02.06 .1996 & 03.06.1996 \\
\hline Weichwerden & & 04.08.1996 & 08.08.1996 & 12.08.1996 & 06.08.1996 & 14.08.1996 & 18.08.1996 & 10.08.1996 & 07.08.1996 \\
\hline $\begin{array}{c}\text { Reife } \\
\left(16^{\circ} \mathrm{Kmw}\right)\end{array}$ & & 12.09.1996 & 02.09.1996 & 09.09.1996 & 07.09.1996 & 09.09.1996 & 09.09.1996 & 09.09.1996 & 05.09.1996 \\
\hline Boden $T>=10^{\circ}$ & \multirow{5}{*}{ बे } & 27.03.1997 & 02.04.1997 & 26.04.1997 & 03.05.1997 & 10.04.1997 & 24.04.1997 & 26.04.1997 & 03.04.1997 \\
\hline Austrieb (7) & & 26.03.1997 & 26.03.1997 & 01.04.1997 & 09.04.1997 & 09.04.1997 & 10.04.1997 & 31.03 .1997 & 02.04.1997 \\
\hline Blühbeginn & & 20.05.1997 & 24.05.1997 & 30.05 .1997 & 31.05 .1997 & 03.06.1997 & 09.06.1997 & 29.05.1997 & 29.05.1997 \\
\hline Weichwerden & & 23.07.1997 & 28.07.1997 & 04.08.1997 & 11.08.1997 & 04.08.1997 & 18.08.1997 & 28.07.1997 & 04.08.1997 \\
\hline $\begin{array}{c}\text { Reife } \\
\left(16^{\circ} \mathrm{Kmw}\right)\end{array}$ & & 24.08.1997 & 26.08.1997 & 01.09.1997 & 03.09.1997 & 02.09.1997 & 09.09.1997 & 31.08 .1997 & 01.09.1997 \\
\hline Boden $T>=10^{\circ}$ & \multirow{5}{*}{$\stackrel{\infty}{\circ}$} & 31.03.1998 & 06.04.1998 & 06.04.1998 & 27.04.1998 & 04.04 .1998 & 23.04.1998 & 23.04.1998 & 04.04 .1998 \\
\hline Austrieb & & 04.04.1998 & 04.04.1998 & 14.04.1998 & 08.04.1998 & 09.04.1998 & 10.04.1998 & 05.04 .1998 & 06.04.1998 \\
\hline Blühbeginn & & 27.05.1998 & 02.06.1998 & 05.06 .1998 & 03.06.1998 & 06.06.1998 & 08.06.1998 & 02.06.1998 & 02.06 .1998 \\
\hline Weichwerden & & 19.07.1998 & 27.07.1998 & 13.08.1998 & 24.07.1998 & 17.08.1998 & 13.08.1998 & 27.07.1998 & 10.08 .1998 \\
\hline $\begin{array}{c}\text { Reife } \\
\left(16^{\circ} \mathrm{Kmw}\right)\end{array}$ & & 17.08.1998 & 24.08.1998 & 31.08.1998 & 30.08 .1998 & 01.09.1998 & 06.09.1998 & 25.08 .1998 & 31.08.1998 \\
\hline Boden $T>=10^{\circ}$ & \multirow{5}{*}{ ஓ్ } & 02.04.1999 & 02.04.1999 & 29.04.1999 & 27.04.1999 & 24.04.1999 & 26.04.1999 & 25.04.1999 & 05.04.1999 \\
\hline Austrieb & & 05.04.1999 & 07.04.1999 & 14.04.1999 & 19.04.1999 & 19.04.1999 & 19.04.1999 & 12.04.1999 & 11.04.1999 \\
\hline Blühbeginn & & 25.05.1999 & 27.05.1999 & 03.06.1999 & 01.06.1999 & 05.06.1999 & 08.06.1999 & 29.05.1999 & 31.05.1999 \\
\hline Weichwerden & & 19.07.1999 & 28.07.1999 & 30.07.1999 & 30.07.1999 & 06.08.1999 & 11.08.1999 & 30.07.1999 & 31.07.1999 \\
\hline $\begin{array}{c}\text { Reife } \\
\left(16^{\circ} \mathrm{Kmw}\right)\end{array}$ & & 23.08.1999 & 27.08.1999 & 06.09.1999 & 06.09.1999 & 05.09.1999 & 10.09.1999 & 31.08 .1999 & 04.09.1999 \\
\hline Boden $T>=10^{\circ}$ & \multirow{5}{*}{ ঠ } & 06.04 .2000 & 06.04 .2000 & 21.04 .2000 & 23.04 .2000 & 19.04 .2000 & 22.04 .2000 & 21.04 .2000 & 19.04.2000 \\
\hline Austrieb & & 18.04 .2000 & 18.04 .2000 & 21.04 .2000 & 22.04 .2000 & 23.04 .2000 & 22.04 .2000 & 22.04 .2000 & 21.04.2000 \\
\hline Blühbeginn & & 22.05 .2000 & 26.05 .2000 & 29.05 .2000 & 28.05 .2000 & 02.06 .2000 & 05.06 .2000 & 29.05 .2000 & 27.05 .2000 \\
\hline Weichwerden & & 17.07 .2000 & 17.07.2000 & 25.07 .2000 & 18.07.2000 & 28.07.2000 & 07.08 .2000 & 21.07.2000 & 19.07.2000 \\
\hline $\begin{array}{c}\text { Reife } \\
\left(16^{\circ} \mathrm{Kmw}\right)\end{array}$ & & 19.08 .2000 & 21.08 .2000 & 28.08 .2000 & 23.08 .2000 & 25.08 .2000 & 30.08 .2000 & 26.08 .2000 & 21.08 .2000 \\
\hline Boden $T>=10^{\circ}$ & \multirow{4}{*}{ ర్రి } & 22.03 .2001 & 25.03.2001 & 29.04.2001 & 29.04 .2001 & 26.04 .2001 & 26.04 .2001 & 27.04.2001 & 27.04.2001 \\
\hline Austrieb & & 29.03.2001 & 06.04.2001 & 12.04 .2001 & 10.04 .2001 & 12.04 .2001 & 20.04 .2001 & 07.04 .2001 & 07.04.2001 \\
\hline Blühbeginn & & 25.05 .2001 & 26.05 .2001 & 01.06 .2001 & 28.05 .2001 & 05.06 .2001 & 08.06.2001 & 28.05 .2001 & 28.05.2001 \\
\hline Weichwerden & & 26.07.2001 & 27.07.2001 & 04.08 .2001 & 30.07 .2001 & 05.08 .2001 & 06.08 .2001 & 27.07.2001 & 31.07 .2001 \\
\hline
\end{tabular}




\begin{tabular}{|c|c|c|c|c|c|c|c|c|c|}
\hline $\begin{array}{c}\text { Reife } \\
\left(16^{\circ} \mathrm{Kmw}\right)\end{array}$ & & 21.08.2001 & 26.08.2001 & 31.08 .2001 & 27.08.2001 & 07.09 .2001 & 08.09.2001 & 26.08.2001 & 06.09 .2001 \\
\hline Boden $\mathrm{T}>=10^{\circ}$ & \multirow{5}{*}{ రి } & 28.03 .2002 & 01.04 .2002 & 23.04 .2002 & 19.04.2002 & 18.04.2002 & 23.04.2002 & 24.04 .2002 & 04.04 .2002 \\
\hline Austrieb & & 08.04 .2002 & 05.04 .2002 & 12.04 .2002 & 19.04.2002 & 19.04 .2002 & 23.04.2002 & 10.04 .2002 & 06.04 .2002 \\
\hline Blühbeginn & & 27.05 .2002 & 31.05 .2002 & 03.06 .2002 & 31.05 .2002 & 10.06 .2002 & 11.06 .2002 & 31.05 .2002 & 31.05 .2002 \\
\hline Weichwerden & & 19.07.2002 & 21.07.2002 & 29.07.2002 & 23.07.2002 & 29.07.2002 & 29.07.2002 & 26.07.2002 & 23.07 .2002 \\
\hline $\begin{array}{c}\text { Reife } \\
\left(16^{\circ} \mathrm{Kmw}\right)\end{array}$ & & - & - & - & - & - & - & - & - \\
\hline
\end{tabular}


Tab. 5: Phenological stages, from 1996 to 2002, at the eight experimental sites in the Überetsch area of South Tyrol.

\begin{tabular}{|c|c|c|c|c|c|c|c|c|c|}
\hline Stadium & & $\begin{array}{l}\text { Kaltern } \\
\text { "St. Josef am } \\
\text { See" }\end{array}$ & $\begin{array}{l}\text { Kaltern } \\
\text { "Mazzon" }\end{array}$ & $\begin{array}{l}\text { Kaltern } \\
\text { "Dorf" }\end{array}$ & $\begin{array}{l}\text { St. Pauls } \\
\text { "Feld" }\end{array}$ & $\begin{array}{l}\text { Planitzing } \\
\text { "Garnel- } \\
\text { len" }\end{array}$ & $\begin{array}{l}\text { Eppan } \\
\text { "Berg" }\end{array}$ & $\begin{array}{l}\text { Girlan } \\
\text { "Doos" }\end{array}$ & $\begin{array}{c}\text { Girlan } \\
\text { "Schreck- } \\
\text { bichl" }\end{array}$ \\
\hline Soil T>= $10^{\circ}$ & \multirow{5}{*}{ बू } & 10.04.1996 & 12.04 .1996 & 20.04.1996 & 23.04.1996 & 18.04.1996 & 21.04.1996 & 20.04.1996 & 19.04.1996 \\
\hline Shooting (7) & & 16.04.1996 & 18.04.1996 & 19.04.1996 & 19.04.1996 & 20.04.1996 & 21.04.1996 & 19.04.1996 & 19.04.1996 \\
\hline $\begin{array}{c}\text { Start of blosso- } \\
\text { ming }\end{array}$ & & 27.05.1996 & 01.06 .1996 & 03.06 .1996 & 01.06 .1996 & 06.06.1996 & 07.06 .1996 & 02.06.1996 & 03.06.1996 \\
\hline Softening & & 04.08.1996 & 08.08 .1996 & 12.08 .1996 & 06.08.1996 & 14.08.1996 & 18.08.1996 & 10.08.1996 & 07.08 .1996 \\
\hline $\begin{array}{l}\text { Ripe (16 } \\
\left.{ }^{\circ} \mathrm{Kmw}\right)\end{array}$ & & 12.09 .1996 & 02.09 .1996 & 09.09.1996 & 07.09.1996 & 09.09.1996 & 09.09.1996 & 09.09.1996 & 05.09.1996 \\
\hline Soil $\mathrm{T}>=10^{\circ}$ & \multirow{5}{*}{ ने } & 27.03.1997 & 02.04 .1997 & 26.04.1997 & 03.05.1997 & 10.04.1997 & 24.04.1997 & 26.04.1997 & 03.04.1997 \\
\hline Shooting (7) & & 26.03.1997 & 26.03.1997 & 01.04.1997 & 09.04.1997 & 09.04.1997 & 10.04.1997 & 31.03.1997 & 02.04.1997 \\
\hline $\begin{array}{c}\text { Start of blosso- } \\
\text { ming }\end{array}$ & & 20.05.1997 & 24.05.1997 & 30.05 .1997 & 31.05 .1997 & 03.06.1997 & 09.06.1997 & 29.05.1997 & 29.05.1997 \\
\hline Softening & & 23.07.1997 & 28.07.1997 & 04.08 .1997 & 11.08.1997 & 04.08.1997 & 18.08.1997 & 28.07.1997 & 04.08 .1997 \\
\hline $\begin{array}{l}\text { Ripe (16 } \\
\left.{ }^{\circ} \mathrm{Kmw}\right)\end{array}$ & & 24.08.1997 & 26.08.1997 & 01.09 .1997 & 03.09.1997 & 02.09.1997 & 09.09.1997 & 31.08.1997 & 01.09.1997 \\
\hline Soil T>= $10^{\circ}$ & \multirow{5}{*}{$\stackrel{\infty}{\text { ने }}$} & 31.03 .1998 & 06.04 .1998 & 06.04 .1998 & 27.04.1998 & 04.04.1998 & 23.04 .1998 & 23.04.1998 & 04.04 .1998 \\
\hline Shooting (7) & & 04.04.1998 & 04.04 .1998 & 14.04 .1998 & 08.04.1998 & 09.04.1998 & 10.04.1998 & 05.04.1998 & 06.04 .1998 \\
\hline $\begin{array}{l}\text { Start of blosso- } \\
\text { ming }\end{array}$ & & 27.05.1998 & 02.06 .1998 & 05.06 .1998 & 03.06.1998 & 06.06.1998 & 08.06 .1998 & 02.06 .1998 & 02.06 .1998 \\
\hline Softening & & 19.07.1998 & 27.07.1998 & 13.08 .1998 & 24.07.1998 & 17.08 .1998 & 13.08 .1998 & 27.07.1998 & 10.08 .1998 \\
\hline $\begin{array}{l}\text { Ripe (16 } \\
\left.{ }^{\circ} \mathrm{Kmw}\right)\end{array}$ & & 17.08 .1998 & 24.08 .1998 & 31.08 .1998 & 30.08 .1998 & 01.09 .1998 & 06.09.1998 & 25.08 .1998 & 31.08 .1998 \\
\hline Soil T $>=10^{\circ}$ & \multirow{5}{*}{ คे } & 02.04.1999 & 02.04.1999 & 29.04.1999 & 27.04.1999 & 24.04.1999 & 26.04.1999 & 25.04.1999 & 05.04.1999 \\
\hline Shooting (7) & & 05.04.1999 & 07.04.1999 & 14.04.1999 & 19.04.1999 & 19.04.1999 & 19.04.1999 & 12.04.1999 & 11.04.1999 \\
\hline $\begin{array}{c}\text { Start of blosso- } \\
\text { ming }\end{array}$ & & 25.05.1999 & 27.05.1999 & 03.06.1999 & 01.06.1999 & 05.06.1999 & 08.06.1999 & 29.05.1999 & 31.05.1999 \\
\hline Softening & & 19.07.1999 & 28.07.1999 & 30.07 .1999 & 30.07.1999 & 06.08.1999 & 11.08.1999 & 30.07.1999 & 31.07 .1999 \\
\hline $\begin{array}{l}\text { Ripe (16 } \\
{ }^{\circ} \mathrm{Kmw} \text { ) }\end{array}$ & & 23.08.1999 & 27.08.1999 & 06.09.1999 & 06.09.1999 & 05.09.1999 & 10.09.1999 & 31.08.1999 & 04.09.1999 \\
\hline Soil $\mathrm{T}>=10^{\circ}$ & \multirow{5}{*}{ ঃ্ণ } & 06.04 .2000 & 06.04 .2000 & 21.04 .2000 & 23.04 .2000 & 19.04.2000 & 22.04 .2000 & 21.04.2000 & 19.04 .2000 \\
\hline Shooting (7) & & 18.04 .2000 & 18.04 .2000 & 21.04 .2000 & 22.04 .2000 & 23.04 .2000 & 22.04 .2000 & 22.04 .2000 & 21.04 .2000 \\
\hline $\begin{array}{c}\text { Start of blosso- } \\
\text { ming }\end{array}$ & & 22.05 .2000 & 26.05 .2000 & 29.05 .2000 & 28.05 .2000 & 02.06 .2000 & 05.06 .2000 & 29.05 .2000 & 27.05 .2000 \\
\hline Softening & & 17.07 .2000 & 17.07 .2000 & 25.07 .2000 & 18.07.2000 & 28.07 .2000 & 07.08 .2000 & 21.07.2000 & 19.07 .2000 \\
\hline $\begin{array}{l}\text { Ripe (16 } \\
\left.{ }^{\circ} \mathrm{Kmw}\right)\end{array}$ & & 19.08 .2000 & 21.08 .2000 & 28.08 .2000 & 23.08 .2000 & 25.08 .2000 & 30.08 .2000 & 26.08 .2000 & 21.08 .2000 \\
\hline Soil T $>=10^{\circ}$ & \multirow{4}{*}{$\ddot{\varnothing}$} & 22.03 .2001 & 25.03 .2001 & 29.04 .2001 & 29.04.2001 & 26.04.2001 & 26.04 .2001 & 27.04.2001 & 27.04.2001 \\
\hline Shooting (7) & & 29.03.2001 & 06.04 .2001 & 12.04 .2001 & 10.04.2001 & 12.04 .2001 & 20.04.2001 & 07.04.2001 & 07.04 .2001 \\
\hline $\begin{array}{l}\text { Start of blosso- } \\
\text { ming }\end{array}$ & & 25.05 .2001 & 26.05 .2001 & 01.06 .2001 & 28.05 .2001 & 05.06 .2001 & 08.06 .2001 & 28.05 .2001 & 28.05.2001 \\
\hline Softening & & 26.07.2001 & 27.07.2001 & 04.08 .2001 & 30.07 .2001 & 05.08 .2001 & 06.08 .2001 & 27.07 .2001 & 31.07.2001 \\
\hline
\end{tabular}




\begin{tabular}{|c|c|c|c|c|c|c|c|c|c|}
\hline $\begin{array}{l}\text { Ripe (16 } \\
\left.{ }^{\circ} \mathrm{Kmw}\right)\end{array}$ & & 21.08.2001 & 26.08.2001 & 31.08 .2001 & 27.08.2001 & 07.09 .2001 & 08.09 .2001 & 26.08.2001 & 06.09 .2001 \\
\hline Soil $T>=10^{\circ}$ & \multirow{5}{*}{ ণิ } & 28.03 .2002 & 01.04 .2002 & 23.04 .2002 & 19.04.2002 & 18.04.2002 & 23.04 .2002 & 24.04 .2002 & 04.04 .2002 \\
\hline Shooting (7) & & 08.04 .2002 & 05.04 .2002 & 12.04 .2002 & 19.04.2002 & 19.04 .2002 & 23.04 .2002 & 10.04 .2002 & 06.04 .2002 \\
\hline $\begin{array}{c}\text { Start of blosso- } \\
\text { ming }\end{array}$ & & 27.05 .2002 & 31.05 .2002 & 03.06 .2002 & 31.05 .2002 & 10.06 .2002 & 11.06 .2002 & 31.05 .2002 & 31.05 .2002 \\
\hline Softening & & 19.07.2002 & 21.07.2002 & 29.07.2002 & 23.07.2002 & 29.07.2002 & 29.07.2002 & 26.07.2002 & 23.07 .2002 \\
\hline $\begin{array}{l}\text { Ripe (16 } \\
\left.{ }^{\circ} \mathrm{Kmw}\right)\end{array}$ & & - & - & - & - & - & - & - & - \\
\hline
\end{tabular}


Tab. 6: Ergebnisse der Varianzanalyse der im Most und Wein gemessenen Parameter (nur Varianten mit unterschiedlichen Buchstaben unterscheiden sich signifikant voneinander, Einfakt. Anova, Tukey-B, Signifikanzniveau 0.05) // Results of variance analysis of the parameters measured in must and wine (only variants with different letters differ significantly from each other, Einfakt. Anova, Tukey-B, Significance level 0.05).

\begin{tabular}{|c|c|c|c|c|c|c|c|c|}
\hline Parameter & $\begin{array}{l}\text { Kaltern } \\
\text { "St. Josef } \\
\text { am See" }\end{array}$ & $\begin{array}{l}\text { Kaltern } \\
\text { "Mazzon" }\end{array}$ & $\begin{array}{l}\text { Kaltern } \\
\text { "Dorf" }\end{array}$ & $\begin{array}{l}\text { Planitzing } \\
\text { "Garnel- } \\
\text { len" }\end{array}$ & $\begin{array}{l}\text { St. Pauls } \\
\text { "Feld" }\end{array}$ & $\begin{array}{l}\text { Eppan } \\
\text { "Berg" }\end{array}$ & $\begin{array}{l}\text { Girlan } \\
\text { "Doos" }\end{array}$ & $\begin{array}{c}\text { Girlan } \\
\text { "Schreck- } \\
\text { bichl" }\end{array}$ \\
\hline $\begin{array}{l}\text { Mostparameter } \\
\text { Must parameters }\end{array}$ & & & & & & & & \\
\hline
\end{tabular}

$\begin{array}{llllllllllllllllll}\mathrm{pH} \text {-Wert } & * * * & 3.57 & \mathrm{c} & 3.43 & \mathrm{~b} & 3.44 & \mathrm{~b} & 3.45 & \mathrm{~b} & 3.49 & \mathrm{bc} & 3.30 & \mathrm{a} & 3.46 & \mathrm{~b} & 3.45\end{array}$

titrierbare Ge-

\begin{tabular}{|c|c|c|c|c|c|c|c|c|c|c|c|c|c|c|c|c|}
\hline $\begin{array}{l}\text { samtsäure }(\mathrm{g} / \mathrm{l}) \\
\text { Titratable acidity } \\
(\mathrm{g} / \mathrm{l})\end{array}$ & $* * *$ & 6.3 & $a b$ & 6.2 & $a b$ & 7.6 & c & 7.5 & c & 6.2 & $a$ & 7.1 & bc & 7.0 & $a b c$ & 6.8 \\
\hline $\begin{array}{l}\text { Mostgewicht } \\
\left({ }^{\circ} \mathrm{KMW}\right) \\
\text { Must weight } \\
\left({ }^{\circ} \mathrm{KMW}\right)\end{array}$ & n.s. & 18.7 & - & 19.1 & - & 18.7 & - & 19.1 & - & 18.6 & - & 19.3 & - & 18.2 & - & 18.9 \\
\hline $\begin{array}{l}\text { Hefeverwertba- } \\
\text { rer Stickstoff } \\
\text { (mg/l) } \\
\text { Yeast assimilable } \\
\text { nitrogen (mg/l) }\end{array}$ & $* * *$ & 210 & $b c$ & 99 & $a b$ & 64 & $a$ & 286 & c & 211 & $\mathrm{bc}$ & 214 & $b c$ & 242 & bc & 180 \\
\hline
\end{tabular}

Weinparameter

Wine parameters

\begin{tabular}{|c|c|c|c|c|c|c|c|c|c|c|c|c|c|c|c|c|c|}
\hline $\begin{array}{l}\text { Weinsäure (g/l) } \\
\text { Tartarci acid (g/l) }\end{array}$ & n.s. & 1.8 & - & 2.2 & - & 2.2 & - & 2.0 & - & 2.3 & - & 2.3 & - & 2.0 & - & 2.1 & - \\
\hline $\begin{array}{l}\text { Milchsäure (g/l) } \\
\text { Lactic acid (g/l) }\end{array}$ & $*$ & 2.8 & $b$ & 2.4 & $a b$ & 2.5 & $a b$ & 2.6 & $a b$ & 2.7 & $a b$ & 2.3 & a & 2.8 & b & 2.7 & $a b$ \\
\hline $\begin{array}{l}\text { pH-Wert } \\
\text { pH value }\end{array}$ & $*$ & 4.02 & $b$ & 3.98 & $a b$ & 3.89 & $a b$ & 3.94 & $a b$ & 3.97 & $a b$ & 3.84 & a & 3.95 & $a b$ & 3.90 & $a b$ \\
\hline $\begin{array}{l}\text { Alkohol (\% Vol.) } \\
\text { Alcohol (\% vol.) }\end{array}$ & $*$ & 12.5 & $a b$ & 12.8 & $a b$ & 12.7 & $a b$ & 12.6 & $a b$ & 12.3 & $a b$ & 13.2 & $b$ & 12.1 & a & 12.6 & $a b$ \\
\hline $\begin{array}{l}\text { Gesamtextrakt } \\
\text { (g/l) } \\
\text { Total dry extract } \\
(\mathrm{g} / \mathrm{l})\end{array}$ & n.s. & 28.6 & - & 28.2 & - & 28.4 & - & 27.2 & - & 28.7 & - & 27.6 & - & 27.7 & - & 28.1 & - \\
\hline $\begin{array}{l}\text { reduzierter Troc- } \\
\text { kenextrakt }(\mathrm{g} / \mathrm{l}) \\
\text { Reduced extract } \\
(\mathrm{g} / \mathrm{l})\end{array}$ & $*$ & 27.19 & & 26.87 & & 27.16 & & 25.97 & & 27.13 & & 26.28 & & 26.48 & & 27.15 & \\
\hline $\begin{array}{l}\text { Restzucker (g/l) } \\
\text { Residual sugars } \\
(\mathrm{g} / \mathrm{l})\end{array}$ & n.s. & 1.9 & - & 2.1 & - & 1.8 & - & 1.8 & - & 2.0 & - & 1.9 & - & 1.7 & - & 1.8 & - \\
\hline
\end{tabular}




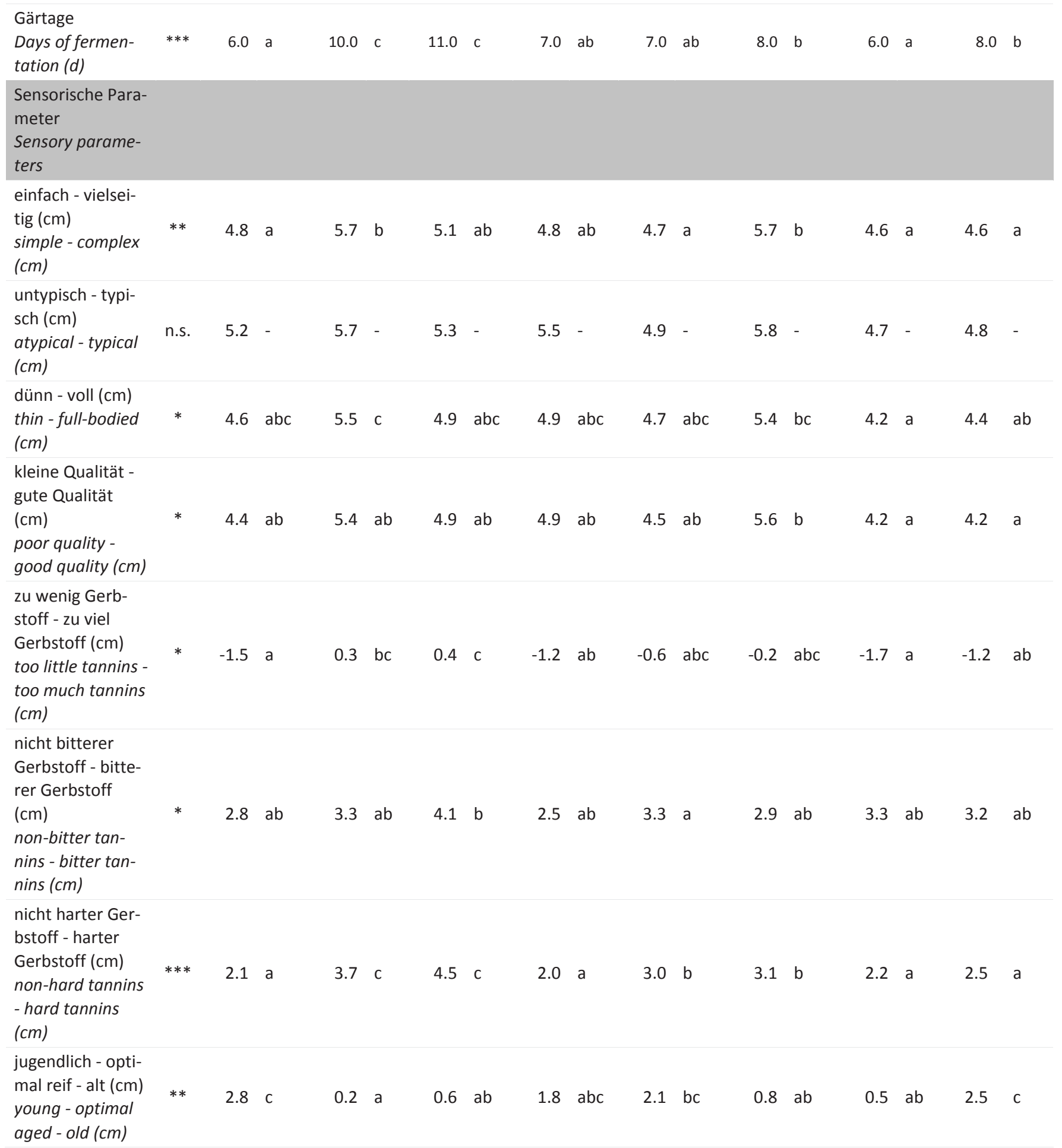


Tab. 7: Signifikante Zusammenhänge zwischen sensorischen Parametern, Most- und Weininhaltsstoffen sowie Bodenkenn- und Klimadaten.

\begin{tabular}{|c|c|c|c|}
\hline Faktor 1 & Faktor 2 & Korr.-koeff. & Sign. \\
\hline \multirow{2}{*}{ Ertrag $\left(\mathrm{kg} / \mathrm{m}^{2}\right)$} & Mittleres Traubengewicht (g) & 0.79 & 0.000 \\
\hline & Verdunstung IV-IX (mm) & 0.61 & 0.000 \\
\hline \multirow{2}{*}{ Mostgewicht ( $\left.{ }^{\circ} \mathrm{KMW}\right)$} & Regen V-VI (mm) & 0.48 & 0.000 \\
\hline & Regen VII-VIII (mm) & -0.48 & 0.000 \\
\hline \multirow{6}{*}{ pH-Wert Most } & Jahresdurchschnittstemperatur $\left({ }^{\circ} \mathrm{C}\right)$ & 0.53 & 0.000 \\
\hline & Verdunstung IV-IX (mm) & 0.51 & 0.000 \\
\hline & Huglin Index & 0.48 & 0.000 \\
\hline & Meereshöhe (m) & -0.51 & 0.000 \\
\hline & titrierbare Gesamtsäure Most (g/l) & -0.51 & 0.000 \\
\hline & Bodentemperatur $\left({ }^{\circ} \mathrm{C}\right)$ & 0.40 & 0.003 \\
\hline \multirow{3}{*}{ titrierbare Gesamtsäure Most (g/l) } & Verdunstung IV-IX (mm) & -0.48 & 0.000 \\
\hline & $\mathrm{pH}$-Wert Most & -0.51 & 0.000 \\
\hline & Jahresdurchschnittstemperatur $\left({ }^{\circ} \mathrm{C}\right)$ & -0.40 & 0.003 \\
\hline \multirow{3}{*}{ Hefeverwertbarer Stickstoff (mg/l) } & Schnittholzgewicht $\left(\mathrm{kg} / \mathrm{m}^{2}\right)$ & 0.72 & 0.000 \\
\hline & Ravaz-Index (kg/kg) & -0.66 & 0.000 \\
\hline & Regen V-VI (mm) & -0.52 & 0.005 \\
\hline \multirow{11}{*}{ pH-Wert Wein } & Regen V-VI (mm) & 0.73 & 0.000 \\
\hline & $\mathrm{pH}$-Wert Most & 0.67 & 0.000 \\
\hline & reduzierter Extrakt (g/l) & 0.66 & 0.000 \\
\hline & Mostgewicht ( $\left.{ }^{\circ} \mathrm{KMW}\right)$ & 0.62 & 0.000 \\
\hline & Jahresdurchschnittstemperatur $\left({ }^{\circ} \mathrm{C}\right)$ & 0.52 & 0.000 \\
\hline & Regen VII-VIII (mm) & -0.42 & 0.000 \\
\hline & titrierbare Gesamtsäure Most (g/l) & -0.44 & 0.000 \\
\hline & titrierbare Gesamtsäure Wein (g/l) & -0.65 & 0.000 \\
\hline & Gesamtpolyphenole (mg/l) & 0.45 & 0.001 \\
\hline & Alkohol (\% Vol.) & 0.43 & 0.001 \\
\hline & Bodentemperatur $\left({ }^{\circ} \mathrm{C}\right)$ & 0.39 & 0.004 \\
\hline \multirow{7}{*}{ Alkohol (\% Vol.) } & Mostgewicht ( $\left.{ }^{\circ} \mathrm{KMW}\right)$ & 0.91 & 0.000 \\
\hline & reduzierter Extrakt (g/l) & 0.64 & 0.000 \\
\hline & Gesamtpolyphenole (mgL) & 0.63 & 0.000 \\
\hline & Milchsäure (g/l) & -0.49 & 0.000 \\
\hline & pH-Wert Wein & 0.43 & 0.001 \\
\hline & Regen VII-VIII (mm) & -0.42 & 0.002 \\
\hline & Ertrag $\left(\mathrm{kg} / \mathrm{m}^{2}\right)$ & -0.52 & 0.005 \\
\hline \multirow{7}{*}{ reduzierter Extrakt (g/l) } & Mostgewicht ( $\left.{ }^{\circ} \mathrm{KMW}\right)$ & 0.69 & 0.000 \\
\hline & pH-Wert Wein & 0.66 & 0.000 \\
\hline & Alkohol (\% Vol.) & 0.64 & 0.000 \\
\hline & Gesamtpolyphenole (mg/l) & 0.61 & 0.000 \\
\hline & Regen V-VI (mm) & 0.49 & 0.000 \\
\hline & Jahresdurchschnittstemperatur $\left({ }^{\circ} \mathrm{C}\right)$ & 0.46 & 0.001 \\
\hline & $\mathrm{pH}$-Wert Most & 0.40 & 0.003 \\
\hline
\end{tabular}


Gesamtpolyphenole (mg/l)

titrierbare Gesamtsäure Wein

Weinsäure (g/l)

Geruchsvielseitigkeit einfach - vielseitig $(\mathrm{cm})$

Typizität

untypisch - typisch (cm)

\begin{tabular}{|c|c|c|}
\hline Gärtage (d) & 0.78 & 0.000 \\
\hline Regen V-VI (mm) & 0.68 & 0.000 \\
\hline Alkohol (\% Vol.) & 0.62 & 0.000 \\
\hline reduzierter Extrakt & 0.61 & 0.000 \\
\hline Mostgewicht ( $\left.{ }^{\circ} \mathrm{KMW}\right)$ & 0.60 & 0.000 \\
\hline Regen VII-VIII (mm) & -0.56 & 0.000 \\
\hline Milchsäure (g/l) & -0.63 & 0.000 \\
\hline Hefeverwertbarer Stickstoff (mg/l) & -0.85 & 0.000 \\
\hline pH-Wert Wein & 0.45 & 0.001 \\
\hline Schnittholzgewicht $\left(\mathrm{kg} / \mathrm{m}^{2}\right)$ & -0.46 & 0.001 \\
\hline Jahresdurchschnittstemperatur $\left({ }^{\circ} \mathrm{C}\right)$ & 0.39 & 0.004 \\
\hline Ertrag $\left(\mathrm{kg} / \mathrm{m}^{2}\right)$ & -0.52 & 0.005 \\
\hline $\mathrm{pH}$-Wert Most & -0.60 & 0.000 \\
\hline pH-Wert Wein & -0.65 & 0.000 \\
\hline Hefeverwertbarer Stickstoff (mg/l) & -0.56 & 0.002 \\
\hline titrierbare Gesamtsäure Most (g/l) & 0.38 & 0.005 \\
\hline Ravaz-Index (kg/kg) & 0.45 & 0.001 \\
\hline Gärtage (d) & 0.43 & 0.001 \\
\hline Hefeverwertbarer Stickstoff (mg/l) & -0.60 & 0.001 \\
\hline Regen VII-VIII (mm) & 0.52 & 0.000 \\
\hline Schnittholzgewicht $\left(\mathrm{kg} / \mathrm{m}^{2}\right)$ & 0.46 & 0.000 \\
\hline Alkohol (\% Vol.) & -0.49 & 0.000 \\
\hline Regen V-VI (mm) & -0.52 & 0.000 \\
\hline Gärtage (d) & -0.57 & 0.000 \\
\hline Gesamtpolyphenole (mg/l) & -0.63 & 0.000 \\
\hline titrierbare Gesamtsäure Most & 0.41 & 0.002 \\
\hline Mostgewicht ( $\left.{ }^{\circ} \mathrm{KMW}\right)$ & -0.41 & 0.002 \\
\hline Hefeverwertbarer Stickstoff (mg/l) & -0.78 & 0.000 \\
\hline kleine Qualität - gute Qualität (cm) & 0.86 & 0.000 \\
\hline untypisch - typisch (cm) & 0.86 & 0.000 \\
\hline dünn - voll (cm) & 0.68 & 0.000 \\
\hline wenig Gerbstoff - viel Gerbstoff (cm) & 0.48 & 0.000 \\
\hline $\mathrm{pH}$-Wert Most & -0.30 & 0.000 \\
\hline jugendlich - alt (cm) & -0.67 & 0.000 \\
\hline Alkohol (\% Vol.) & 0.43 & 0.001 \\
\hline Gesamtpolyphenole (mg/l) & 0.27 & 0.002 \\
\hline Meereshöhe (m) & 0.23 & 0.003 \\
\hline Huglin Index & -0.24 & 0.005 \\
\hline kleine Qualität - gute Qualität (cm) & 0.88 & 0.000 \\
\hline Geruch einfach - vielseitig (cm) & 0.85 & 0.000 \\
\hline dünn - voll (cm) & 0.72 & 0.000 \\
\hline wenig Gerbstoff - viel Gerbstoff (cm) & 0.42 & 0.000 \\
\hline Alkohol (\% Vol.) & 0.34 & 0.000 \\
\hline jugendlich - alt (cm) & -0.52 & 0.000 \\
\hline
\end{tabular}


Fülle

dünn - voll (cm)

Gesamteindruck

kleine Qualität - gute Qualität (cm)
Gesamtpolyphenole (mg/l)

0.26

Huglin Index

kleine Qualität - gute Qualität (cm)

Typizität untypisch - typisch $(\mathrm{cm})$

Geruch einfach - vielseitig $(\mathrm{cm})$

wenig Gerbstoff - viel Gerbstoff (cm)

Alkohol (\% Vol.)

Gesamtpolyphenole (mg/l)

Mostgewicht ( ${ }^{\circ} \mathrm{KMW}$ )

Regen V-VI (mm)

Schnittholzgewicht $\left(\mathrm{kg} / \mathrm{m}^{2}\right)$

Regen VII-VIII (mm)

Milchsäure (g/l)

jugendlich - alt $(\mathrm{cm})$

Meereshöhe $(\mathrm{m})$

Hefeverwertbarer Stickstoff (mg/l)

Weinsäure (g/l)

Gerbstoff hart - weich (cm)

Typizität untypisch - typisch $(\mathrm{cm})$

Geruch einfach - vielseitig $(\mathrm{cm})$

dünn - voll (cm)

wenig Gerbstoff - viel Gerbstoff (cm)

Alkohol (\% Vol.)

Gesamtpolyphenole (mg/l)

Meereshöhe (m)

Mostgewicht ( $\left.{ }^{\circ} \mathrm{KMW}\right)$

Milchsäure (g/l)

jugendlich - alt $(\mathrm{cm})$

Huglin Index

Schnittholzgewicht $\left(\mathrm{kg} / \mathrm{m}^{2}\right)$

Durchschnittstemperatur IV-IX $\left({ }^{\circ} \mathrm{C}\right)$

pH-Wert Most

Regen VII-VIII (mm)

Regen V-VI (mm)

Gerbstoff hart - weich (cm)

dünn - voll (cm)

Gesamtpolyphenole (mg/l)

kleine Qualität - gute Qualität (cm)

Geruch einfach - vielseitig $(\mathrm{cm})$

Gerbstoff bitter - nicht bitter (cm)

Typizität untypisch - typisch (cm)

Weinsäure (g/l)

titrierbare Gesamtsäure Wein (g/l)

Ravaz-Index (kg/kg)

\section{$-0.24$}

0.86

0.72

0.68

0.62

0.53

0.48

0.41

0.32

$-0.31$

$-0.33$

$-0.45$

$-0.52$

0.27

$-0.40$

0.25

0.25

0.88

0.86

0.86

0.58

0.44

0.36

0.31

0.30

$-0.35$

$-0.63$

$-0.29$

$-0.27$

$-0.27$

$-0.25$

$-0.26$

0.24

0.68

0.62

0.61

0.58

0.48

0.44

0.42

0.42

0.40

0.33
0.003

0.005

0.000

0.000

0.000

0.000

0.000

0.000

0.000

0.000

0.000

0.000

0.000

0.000

0.002

0.002

0.004

0.004

0.000

0.000

0.000

0.000

0.000

0.000

0.000

0.000

0.000

0.000

0.001

0.002

0.002

0.003

0.003

0.005

0.000

0.000

0.000

0.000

0.000

0.000

0.000

0.000

0.000

0.000 
Gerbstoff

bitter - nicht bitter (cm)

Gerbstoff

hart - weich $(\mathrm{cm})$

Entwicklungszustand jugendlich - alt $(\mathrm{cm})$

\begin{tabular}{|c|c|c|}
\hline Alkohol (\% Vol.) & 0.30 & 0.000 \\
\hline Regen V-VI (mm) & 0.30 & 0.000 \\
\hline Schnittholzgewicht $\left(\mathrm{kg} / \mathrm{m}^{2}\right)$ & -0.45 & 0.000 \\
\hline Milchsäure (g/l) & -0.47 & 0.000 \\
\hline jugendlich - alt (cm) & -0.64 & 0.000 \\
\hline Hefeverwertbarer Stickstoff (mg/l) & -0.74 & 0.000 \\
\hline Meereshöhe (m) & 0.29 & 0.001 \\
\hline Regen VII-VIII (mm) & -0.24 & 0.005 \\
\hline Gerbstoff hart - weich (cm) & 0.67 & 0.000 \\
\hline Gesamtpoyphenole (mg/l) & 0.51 & 0.000 \\
\hline wenig Gerbstoff - viel Gerbstoff (cm) & 0.44 & 0.000 \\
\hline Regen V-VI (mm) & 0.34 & 0.000 \\
\hline Jahresdurchschnittstemperatur $\left({ }^{\circ} \mathrm{C}\right)$ & 0.33 & 0.000 \\
\hline Milchsäure (g/l) & -0.30 & 0.000 \\
\hline Hefeverwertbarer Stickstoff (mg/l) & -0.55 & 0.000 \\
\hline reduzierter Extrakt (g/l) & 0.26 & 0.002 \\
\hline Regen VII-VIII (mm) & -0.27 & 0.002 \\
\hline titrierbare Gesamtsäure Wein (g/l) & 0.25 & 0.004 \\
\hline Schnittholzgewicht $\left(\mathrm{kg} / \mathrm{m}^{2}\right)$ & -0.25 & 0.004 \\
\hline wenig Gerbstoff - viel Gerbstoff (cm) & 0.68 & 0.000 \\
\hline Gerbstoff bitter - nicht bitter $(\mathrm{cm})$ & 0.67 & 0.000 \\
\hline Gesamtpoyphenole (mg/l) & 0.63 & 0.000 \\
\hline titrierbare Gesamtsäure Wein (g/l) & 0.41 & 0.000 \\
\hline Regen V-VI (mm) & 0.35 & 0.000 \\
\hline Weinsäure (g/l) & 0.35 & 0.000 \\
\hline Ravaz-Index (kg/kg) & 0.35 & 0.000 \\
\hline Jahresdurchschnittstemperatur $\left({ }^{\circ} \mathrm{C}\right)$ & 0.25 & 0.000 \\
\hline Regen VII-VIII (mm) & -0.31 & 0.000 \\
\hline Schnittholzgewicht $\left(\mathrm{kg} / \mathrm{m}^{2}\right)$ & -0.45 & 0.000 \\
\hline Milchsäure (g/l) & -0.46 & 0.000 \\
\hline jugendlich - alt (cm) & -0.51 & 0.000 \\
\hline Hefeverwertbarer Stickstoff (mg/l) & -0.73 & 0.000 \\
\hline dünn - voll (cm) & 0.25 & 0.004 \\
\hline Hefeverwertbarer Stickstoff (mg/l) & 0.60 & 0.000 \\
\hline Schnittholzgewicht $\left(\mathrm{kg} / \mathrm{m}^{2}\right)$ & 0.40 & 0.000 \\
\hline Milchsäure (g/l) & 0.38 & 0.000 \\
\hline $\mathrm{pH}$-Wert Most & 0.35 & 0.000 \\
\hline Meereshöhe (m) & -0.32 & 0.000 \\
\hline Gesamtpolyphenole (mg/l) & -0.36 & 0.000 \\
\hline Ravaz-Index (kg/kg) & -0.36 & 0.000 \\
\hline titrierbare Gesamtsäure Wein (g/l) & -0.43 & 0.000 \\
\hline Gerbstoff hart - weich (cm) & -0.51 & 0.000 \\
\hline dünn - voll (cm) & -0.52 & 0.000 \\
\hline Typizität untypisch - typisch (cm) & -0.52 & 0.000 \\
\hline
\end{tabular}


kleine Qualität - gute Qualität (cm)

wenig Gerbstoff - viel Gerbstoff (cm)

Geruch einfach - vielseitig $(\mathrm{cm})$

Weinsäure (g/l)
$-0.63$

0.000

$-0.64$

0.000

$-0.67$

0.000

$-0.29$

0.001 
Tab. 5: Significant correlations between sensory parameters, must and wine contents, and soil and climate data.

\begin{tabular}{|c|c|c|c|}
\hline Factor 1 & Factor 2 & Coeff. of corr. & Sign. \\
\hline \multirow{2}{*}{ Yield $\left(\mathrm{kg} / \mathrm{m}^{2}\right)$} & Mean berry weight (g) & 0.79 & 0.000 \\
\hline & Evaporation IV-IX (mm) & 0.61 & 0.000 \\
\hline \multirow{2}{*}{ Must weight ( $\left.{ }^{\circ} \mathrm{KMW}\right)$} & Rain V-VI (mm) & 0.48 & 0.000 \\
\hline & Rain VII-VIII (mm) & -0.48 & 0.000 \\
\hline \multirow{6}{*}{$\mathrm{pH}$-value of must } & Average annual temperature $\left({ }^{\circ} \mathrm{C}\right)$ & 0.53 & 0.000 \\
\hline & Evaporation IV-IX (mm) & 0.51 & 0.000 \\
\hline & Huglin Index & 0.48 & 0.000 \\
\hline & Elevation (m) & -0.51 & 0.000 \\
\hline & Titratable acidity of must (g/l) & -0.51 & 0.000 \\
\hline & Soil temperature $\left({ }^{\circ} \mathrm{C}\right)$ & 0.40 & 0.003 \\
\hline \multirow{3}{*}{ Titratable acidity of must (g/l) } & Evaporation IV-IX (mm) & -0.48 & 0.000 \\
\hline & $\mathrm{pH}$-value of must & -0.51 & 0.000 \\
\hline & Average annual temperature $\left({ }^{\circ} \mathrm{C}\right)$ & -0.40 & 0.003 \\
\hline \multirow{3}{*}{ Yeast assimilable nitrogen $(\mathrm{mg} / \mathrm{l})$} & Pruned wood weight $\left(\mathrm{kg} / \mathrm{m}^{2}\right)$ & 0.72 & 0.000 \\
\hline & Ravaz Index (kg/kg) & -0.66 & 0.000 \\
\hline & Rain V-VI (mm) & -0.52 & 0.005 \\
\hline \multirow{11}{*}{$\mathrm{pH}$-value of wine } & Rain V-VI (mm) & 0.73 & 0.000 \\
\hline & $\mathrm{pH}$-value of must & 0.67 & 0.000 \\
\hline & Reduced extract $(\mathrm{g} / \mathrm{l})$ & 0.66 & 0.000 \\
\hline & Must weight ('KMW) & 0.62 & 0.000 \\
\hline & Average annual temperature $\left({ }^{\circ} \mathrm{C}\right)$ & 0.52 & 0.000 \\
\hline & Rain VII-VIII (mm) & -0.42 & 0.000 \\
\hline & Titratable acidity of must (g/l) & -0.44 & 0.000 \\
\hline & Titratable acidity of wine $(\mathrm{g} / \mathrm{l})$ & -0.65 & 0.000 \\
\hline & Total polyphenols (mg/l) & 0.45 & 0.001 \\
\hline & Alcohol (\% by vol.) & 0.43 & 0.001 \\
\hline & Soil temperature $\left({ }^{\circ} \mathrm{C}\right)$ & 0.39 & 0.004 \\
\hline \multirow{7}{*}{ Alcohol (\% by vol.) } & Must weight ('KMW) & 0.91 & 0.000 \\
\hline & Reduced extract $(\mathrm{g} / \mathrm{l})$ & 0.64 & 0.000 \\
\hline & Total polyphenols (mgL) & 0.63 & 0.000 \\
\hline & Lactic acid (g/l) & -0.49 & 0.000 \\
\hline & $\mathrm{pH}$-value of wine & 0.43 & 0.001 \\
\hline & Rain VII-VIII (mm) & -0.42 & 0.002 \\
\hline & Yield $\left(\mathrm{kg} / \mathrm{m}^{2}\right)$ & -0.52 & 0.005 \\
\hline \multirow{7}{*}{ Reduced extract $(\mathrm{g} / \mathrm{l})$} & Must weight (('KMW) & 0.69 & 0.000 \\
\hline & $\mathrm{pH}$-value of wine & 0.66 & 0.000 \\
\hline & Alcohol (\% by vol.) & 0.64 & 0.000 \\
\hline & Total polyphenols (mg/l) & 0.61 & 0.000 \\
\hline & Rain V-VI (mm) & 0.49 & 0.000 \\
\hline & Average annual temperature $\left({ }^{\circ} \mathrm{C}\right)$ & 0.46 & 0.001 \\
\hline & $\mathrm{pH}$-value of must & 0.40 & 0.003 \\
\hline
\end{tabular}


Total polyphenols (mg/l)

Titratable total acid of wine

Tartaric acid (g/l)

Complexity of bouquet simple - complex $(\mathrm{cm})$

Typicality

atypical - typical $(\mathrm{cm})$

\begin{tabular}{|c|c|c|}
\hline Days of fermentation (d) & 0.78 & 0.000 \\
\hline Rain V-VI (mm) & 0.68 & 0.000 \\
\hline Alcohol (\% by vol.) & 0.62 & 0.000 \\
\hline Reduced extract & 0.61 & 0.000 \\
\hline Must weight ( $\left.{ }^{\circ} \mathrm{KMW}\right)$ & 0.60 & 0.000 \\
\hline Rain VII-VIII (mm) & -0.56 & 0.000 \\
\hline Lactic acid (g/l) & -0.63 & 0.000 \\
\hline Yeast assimilable nitrogen (mg/l) & -0.85 & 0.000 \\
\hline $\mathrm{pH}$-value of wine & 0.45 & 0.001 \\
\hline Pruned wood weight $\left(\mathrm{kg} / \mathrm{m}^{2}\right)$ & -0.46 & 0.001 \\
\hline Average annual temperature $\left({ }^{\circ} \mathrm{C}\right)$ & 0.39 & 0.004 \\
\hline Yield $\left(\mathrm{kg} / \mathrm{m}^{2}\right)$ & -0.52 & 0.005 \\
\hline $\mathrm{pH}$-value of must & -0.60 & 0.000 \\
\hline $\mathrm{pH}$-value of wine & -0.65 & 0.000 \\
\hline Yeast assimilable nitrogen (mg/l) & -0.56 & 0.002 \\
\hline Titratable acidity of must (g/l) & 0.38 & 0.005 \\
\hline Ravaz Index (kg/kg) & 0.45 & 0.001 \\
\hline Days of fermentation (d) & 0.43 & 0.001 \\
\hline Yeast assimilable nitrogen (mg/l) & -0.60 & 0.001 \\
\hline Rain VII-VIII (mm) & 0.52 & 0.000 \\
\hline Pruned wood weight $\left(\mathrm{kg} / \mathrm{m}^{2}\right)$ & 0.46 & 0.000 \\
\hline Alcohol (\% by vol.) & -0.49 & 0.000 \\
\hline Rain V-VI (mm) & -0.52 & 0.000 \\
\hline Days of fermentation (d) & -0.57 & 0.000 \\
\hline Total polyphenols (mg/l) & -0.63 & 0.000 \\
\hline Titratable total acid of must & 0.41 & 0.002 \\
\hline Must weight ( $\left.{ }^{\circ} \mathrm{KMW}\right)$ & -0.41 & 0.002 \\
\hline Yeast assimilable nitrogen (mg/l) & -0.78 & 0.000 \\
\hline Poor quality - good quality(cm) & 0.86 & 0.000 \\
\hline Atypical - typical (cm) & 0.86 & 0.000 \\
\hline Thin - full-bodied (cm) & 0.68 & 0.000 \\
\hline Too little tannins - too much tannins $(\mathrm{cm})$ & 0.48 & 0.000 \\
\hline $\mathrm{pH}$-value of must & -0.30 & 0.000 \\
\hline Young - old (cm) & -0.67 & 0.000 \\
\hline Alcohol (\% by vol.) & 0.43 & 0.001 \\
\hline Total polyphenols (mg/l) & 0.27 & 0.002 \\
\hline Elevation (m) & 0.23 & 0.003 \\
\hline Huglin Index & -0.24 & 0.005 \\
\hline Poor quality - good quality $(\mathrm{cm})$ & 0.88 & 0.000 \\
\hline Bouquet, simple - complex (cm) & 0.85 & 0.000 \\
\hline Thin - full-bodied (cm) & 0.72 & 0.000 \\
\hline Too little tannins - too much tannins $(\mathrm{cm})$ & 0.42 & 0.000 \\
\hline Alcohol (\% by vol.) & 0.34 & 0.000 \\
\hline Young - old (cm) & -0.52 & 0.000 \\
\hline
\end{tabular}


Full-bodiedness

thin - full-bodied (cm)

Overall impression

poor quality - good quality $(\mathrm{cm}$ )

Tannins (sensory)

too little - too much $(\mathrm{cm})$

\begin{tabular}{|c|c|c|}
\hline Total polyphenols (mg/l) & 0.26 & 0.003 \\
\hline Huglin Index & -0.24 & 0.005 \\
\hline Poor quality - good quality(cm) & 0.86 & 0.000 \\
\hline Typicality, atypical - typical (cm) & 0.72 & 0.000 \\
\hline Bouquet, simple - complex (cm) & 0.68 & 0.000 \\
\hline Too little tannins - too much tannins $(\mathrm{cm})$ & 0.62 & 0.000 \\
\hline Alcohol (\% by vol.) & 0.53 & 0.000 \\
\hline Total polyphenols (mg/l) & 0.48 & 0.000 \\
\hline Must weight ( $\left.{ }^{\circ} \mathrm{KMW}\right)$ & 0.41 & 0.000 \\
\hline Rain V-VI (mm) & 0.32 & 0.000 \\
\hline Pruned wood weight $\left(\mathrm{kg} / \mathrm{m}^{2}\right)$ & -0.31 & 0.000 \\
\hline Rain VII-VIII (mm) & -0.33 & 0.000 \\
\hline Lactic acid (g/l) & -0.45 & 0.000 \\
\hline Young - old (cm) & -0.52 & 0.000 \\
\hline Elevation (m) & 0.27 & 0.002 \\
\hline Yeast assimilable nitrogen (mg/l) & -0.40 & 0.002 \\
\hline Tartaric acid (g/l) & 0.25 & 0.004 \\
\hline Tannins, hard - soft $(\mathrm{cm})$ & 0.25 & 0.004 \\
\hline Typicality, atypical - typical (cm) & 0.88 & 0.000 \\
\hline Bouquet, simple - complex (cm) & 0.86 & 0.000 \\
\hline Thin - full-bodied (cm) & 0.86 & 0.000 \\
\hline Too little tannins - too much tannins $(\mathrm{cm})$ & 0.58 & 0.000 \\
\hline Alcohol (\% by vol.) & 0.44 & 0.000 \\
\hline Total polyphenols (mg/l) & 0.36 & 0.000 \\
\hline Elevation (m) & 0.31 & 0.000 \\
\hline Must weight ( $\left.{ }^{\circ} \mathrm{KMW}\right)$ & 0.30 & 0.000 \\
\hline Lactic acid (g/l) & -0.35 & 0.000 \\
\hline Young - old (cm) & -0.63 & 0.000 \\
\hline Huglin Index & -0.29 & 0.001 \\
\hline Pruned wood weight $\left(\mathrm{kg} / \mathrm{m}^{2}\right)$ & -0.27 & 0.002 \\
\hline Average temperature IV-IX $\left({ }^{\circ} \mathrm{C}\right)$ & -0.27 & 0.002 \\
\hline $\mathrm{pH}$-value of must & -0.25 & 0.003 \\
\hline Rain VII-VIII (mm) & -0.26 & 0.003 \\
\hline Rain V-VI (mm) & 0.24 & 0.005 \\
\hline Tannins, hard - soft (cm) & 0.68 & 0.000 \\
\hline Thin - full-bodied $(\mathrm{cm})$ & 0.62 & 0.000 \\
\hline Total polyphenols (mg/l) & 0.61 & 0.000 \\
\hline Poor quality - good quality $(\mathrm{cm})$ & 0.58 & 0.000 \\
\hline Bouquet, simple - complex (cm) & 0.48 & 0.000 \\
\hline Tannins, bitter - not bitter (cm) & 0.44 & 0.000 \\
\hline Typicality, atypical - typical (cm) & 0.42 & 0.000 \\
\hline Tartaric acid (g/l) & 0.42 & 0.000 \\
\hline Titratable acidity of wine $(\mathrm{g} / \mathrm{l})$ & 0.40 & 0.000 \\
\hline Ravaz Index (kg/kg) & 0.33 & 0.000 \\
\hline
\end{tabular}


Tannins

bitter - not bitter $(\mathrm{cm})$

Tannins

hard - soft $(\mathrm{cm})$

Developmental stage

young - old $(\mathrm{cm})$

\begin{tabular}{|c|c|c|}
\hline Alcohol (\% by vol.) & 0.30 & 0.000 \\
\hline Rain V-VI (mm) & 0.30 & 0.000 \\
\hline Pruned wood weight $\left(\mathrm{kg} / \mathrm{m}^{2}\right)$ & -0.45 & 0.000 \\
\hline Lactic acid (g/l) & -0.47 & 0.000 \\
\hline Young - old (cm) & -0.64 & 0.000 \\
\hline Yeast assimilable nitrogen (mg/l) & -0.74 & 0.000 \\
\hline Elevation (m) & 0.29 & 0.001 \\
\hline Rain VII-VIII (mm) & -0.24 & 0.005 \\
\hline Tannins, hard - soft (cm) & 0.67 & 0.000 \\
\hline Total polyphenols (mg/l) & 0.51 & 0.000 \\
\hline Too little tannins - too much tannins $(\mathrm{cm})$ & 0.44 & 0.000 \\
\hline Rain V-VI (mm) & 0.34 & 0.000 \\
\hline Average annual temperature $\left({ }^{\circ} \mathrm{C}\right)$ & 0.33 & 0.000 \\
\hline Lactic acid (g/l) & -0.30 & 0.000 \\
\hline Yeast assimilable nitrogen (mg/l) & -0.55 & 0.000 \\
\hline Reduced extract $(\mathrm{g} / \mathrm{l})$ & 0.26 & 0.002 \\
\hline Rain VII-VIII (mm) & -0.27 & 0.002 \\
\hline Titratable acidity of wine $(\mathrm{g} / \mathrm{l})$ & 0.25 & 0.004 \\
\hline Pruned wood weight $\left(\mathrm{kg} / \mathrm{m}^{2}\right)$ & -0.25 & 0.004 \\
\hline Too little tannins - too much tannins $(\mathrm{cm})$ & 0.68 & 0.000 \\
\hline Tannins, bitter - not bitter (cm) & 0.67 & 0.000 \\
\hline Total polyphenols (mg/l) & 0.63 & 0.000 \\
\hline Titratable acidity of wine $(\mathrm{g} / \mathrm{l})$ & 0.41 & 0.000 \\
\hline Rain V-VI (mm) & 0.35 & 0.000 \\
\hline Tartaric acid (g/l) & 0.35 & 0.000 \\
\hline Ravaz Index (kg/kg) & 0.35 & 0.000 \\
\hline Average annual temperature $\left({ }^{\circ} \mathrm{C}\right)$ & 0.25 & 0.000 \\
\hline Rain VII-VIII (mm) & -0.31 & 0.000 \\
\hline Pruned wood weight $\left(\mathrm{kg} / \mathrm{m}^{2}\right)$ & -0.45 & 0.000 \\
\hline Lactic acid (g/l) & -0.46 & 0.000 \\
\hline Young - old (cm) & -0.51 & 0.000 \\
\hline Yeast assimilable nitrogen (mg/l) & -0.73 & 0.000 \\
\hline Thin - full-bodied (cm) & 0.25 & 0.004 \\
\hline Yeast assimilable nitrogen (mg/l) & 0.60 & 0.000 \\
\hline Pruned wood weight $\left(\mathrm{kg} / \mathrm{m}^{2}\right)$ & 0.40 & 0.000 \\
\hline Lactic acid (g/l) & 0.38 & 0.000 \\
\hline $\mathrm{pH}$-value of must & 0.35 & 0.000 \\
\hline Elevation (m) & -0.32 & 0.000 \\
\hline Total polyphenols (mg/l) & -0.36 & 0.000 \\
\hline Ravaz Index (kg/kg) & -0.36 & 0.000 \\
\hline Titratable acidity of wine $(\mathrm{g} / \mathrm{l})$ & -0.43 & 0.000 \\
\hline Tannins, hard - soft (cm) & -0.51 & 0.000 \\
\hline Thin - full-bodied (cm) & -0.52 & 0.000 \\
\hline Typicality, atypical - typical (cm) & -0.52 & 0.000 \\
\hline
\end{tabular}




$\begin{array}{lcc}\text { Poor quality - good quality }(\mathrm{cm}) & -0.63 & 0.000 \\ \text { Too little tannins - too much tannins }(\mathrm{cm}) & -0.64 & 0.000 \\ \text { Bouquet, simple - complex }(\mathrm{cm}) & -0.67 & 0.000 \\ \text { Tartaric acid }(\mathrm{g} / \mathrm{l}) & -0.29 & 0.001\end{array}$

\section{(c) $(1) \Theta$}

Dieses Werk ist lizenziert unter einer Creative Commons Namensnennung-Nicht kommerziell 4.0 International Lizenz. Quest'opera è distribuita con Licenza Creative Commons Attribuzione - Non commerciale 4.0 Internazionale. This work is licensed under a Creative Commons Attribution-NonCommercial 4.0 International License.

Für alle Abbildungen und Tabellen ohne Nennung des Urhebers gilt: (C) Versuchszentrum Laimburg.

Per tutte le immagini e tabelle senza menzione dell'artefice vale: (c) Centro di Sperimentazione Laimburg.

For all figures and tables without mention of the originator applies: (C) Laimburg Research Centre. 